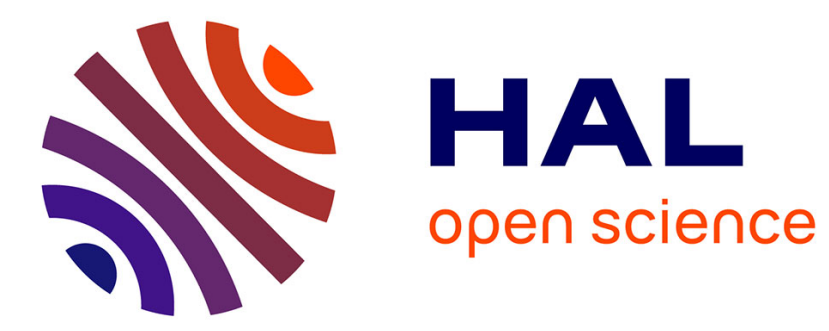

\title{
Global mode interaction and pattern selection in the wake of a disk: A weakly nonlinear expansion
}

Philippe Meliga, Jean-Marc Chomaz, Denis Sipp

\section{To cite this version:}

Philippe Meliga, Jean-Marc Chomaz, Denis Sipp. Global mode interaction and pattern selection in the wake of a disk: A weakly nonlinear expansion. Journal of Fluid Mechanics, 2009, 633 (Août), pp.159-189. 10.1017/s0022112009007290 . hal-01002592

\section{HAL Id: hal-01002592}

https://hal-polytechnique.archives-ouvertes.fr/hal-01002592

Submitted on 7 Jul 2014

HAL is a multi-disciplinary open access archive for the deposit and dissemination of scientific research documents, whether they are published or not. The documents may come from teaching and research institutions in France or abroad, or from public or private research centers.
L'archive ouverte pluridisciplinaire HAL, est destinée au dépôt et à la diffusion de documents scientifiques de niveau recherche, publiés ou non, émanant des établissements d'enseignement et de recherche français ou étrangers, des laboratoires publics ou privés. 


\title{
Global mode interaction and pattern selection in the wake of a disk: a weakly nonlinear expansion
}

\author{
PHILIPPE MELIGA ${ }^{1} \dagger$, JEAN-MARC CHOMAZ $Z^{1,2}$ \\ AND DENIS SIP P \\ ${ }^{1}$ ONERA/DAFE, 8 rue des Vertugadins, 92190 Meudon, France \\ ${ }^{2}$ LadHyX, CNRS-Ecole Polytechnique, 91128 Palaiseau, France
}

(Received 17 July 2008 and in revised form 19 February 2009)

Direct numerical simulations (DNS) of the wake of a circular disk placed normal to a uniform flow show that, as the Reynolds number is increased, the flow undergoes a sequence of successive bifurcations, each state being characterized by specific time and space symmetry breaking or recovering (Fabre, Auguste \& Magnaudet, Phys. Fluids, vol. 20 (5), 2008, p. 1). To explain this bifurcation scenario, we investigate the stability of the axisymmetric steady wake in the framework of the global stability theory. Both the direct and adjoint eigenvalue problems are solved. The threshold Reynolds numbers $R e$ and characteristics of the destabilizing modes agree with the study of Natarajan \& Acrivos (J. Fluid Mech., vol. 254, 1993, p. 323): the first destabilization occurs for a stationary mode of azimuthal wavenumber $m=1$ at $R e_{c}^{A}=116.9$, and the second destabilization of the axisymmetric flow occurs for two oscillating modes of azimuthal wavenumbers $m \pm 1$ at $R e_{c}^{B}=125.3$. Since these critical Reynolds numbers are close to one another, we use a multiple time scale expansion to compute analytically the leading-order equations that describe the nonlinear interaction of these three leading eigenmodes. This set of equations is given by imposing, at third order in the expansion, a Fredholm alternative to avoid any secular term. It turns out to be identical to the normal form predicted by symmetry arguments. Though, all coefficients of the normal form are here analytically computed as the scalar product of an adjoint global mode with a resonant third-order forcing term, arising from the second-order base flow modification and harmonics generation. We show that all nonlinear interactions between modes take place in the recirculation bubble, as the contribution to the scalar product of regions located outside the recirculation bubble is zero. The normal form accurately predicts the sequence of bifurcations, the associated thresholds and symmetry properties observed in the DNS calculations.

\section{Introduction}

When a steady flow loses its stability owing to the variation of a control parameter, it bifurcates towards a new state, that may be either steady or unsteady. If the bifurcation is supercritical and a single eigenmode is responsible for the instability, the dynamics close to the threshold will occur in the one-dimensional slow manifold spanned by the destabilizing eigenmode. The only degree of freedom is then the

$\dagger$ Email address for correspondence: philippe.meliga@ladhyx.polytechnique.fr 
amplitude along the unstable eigenmode direction, which is ruled by a first-order differential equation of polynomial form, the normal form. When several eigenmodes are concomitantly responsible for the destabilization of the steady state, the same reasoning holds, the dimension of the slow manifold being then equal to the number of bifurcating modes, and the normal form involves one degree of freedom per bifurcating mode. Such cases are known as multiple codimension bifurcations, and usually require several independent parameters to be tuned for the different modes to be simultaneously neutral. The normal form then describes the nonlinear interactions between each bifurcating mode and reduces the dynamics of the whole system to a low-dimensional model. For codimension larger than one, the normal form may successfully predict complex behaviours (Crawford \& Knobloch 1991). The analysis requires the following steps to be achieved:

(i) resolution of the linear stability problem to identify the marginally stable modes,

(ii) computation of the nonlinear terms governing the time-asymptotic evolution of these modes and

(iii) truncation of the system at some given order and analysis of the resulting dynamics.

In many physical problems, the structure of the normal form may be directly deduced from phase and symmetry considerations, and multiple codimension bifurcation theory has been successfully used to unravel complex bifurcation structures (Golubitsky \& Stewart 1985; Cross 1986; Crawford, Golubitsky \& Langford 1988; Golubitsky \& Langford 1988; Chossat \& Iooss 1994). However, these problems were involving a base state inhomogeneous only in a single direction, making the expansion procedure tractable. Wakes, and more generally open flows are more complex since the base flow is usually strongly non-parallel. Analysing their stability thus requires to consider modes that are inhomogeneous in both the cross-stream and the streamwise directions, called global modes in reference to their specific streamwise structure, first introduced by Jackson (1987) and Zebib (1987) (see Chomaz 2005, for a review).

The flow past a circular cylinder is a simple example of codimension one bifurcation, where the steady flow is destabilized by an oscillating global mode at the Reynolds number $R e=47$ (Ding \& Kawahara 1999; Barkley 2006). The flow undergoes a Hopf bifurcation, and the normal form reduces to the Stuart-Landau amplitude equation that reads

$$
\dot{A}=\lambda A-\mu A|A|^{2},
$$

where $A$ is the amplitude of the bifurcating mode and $\lambda$ its linear complex growth rate. In this case, the complex Landau coefficient $\mu$ has been computed by fitting methods from experimental measurements (Provansal, Mathis \& Boyer 1987) or from direct numerical simulations (DNS) (Dušek, Le Gal \& Fraunie 1994). In this context, the Landau coefficient is relative to the particular point in space where the experimental or numerical signal is extracted, and to the particular variable that is indeed measured. Recently, the Landau coefficient has been obtained by a standard weakly nonlinear stability analysis based on the bifurcating global mode (Sipp \& Lebedev 2007), which showed that the nonlinear self-interaction was mainly acting through base flow modifications and not through harmonics generation.

We consider here the wake past a flat circular disk of diameter $D$, placed orthogonally with respect to a uniform flow of velocity $U_{\infty}$, at low Reynolds numbers $\operatorname{Re}<150$, where $\operatorname{Re}=D U_{\infty} / \nu$ and $v$ is the kinematic viscosity of the fluid. This problem has been recently addressed using DNS calculations by Fabre, Auguste \& 
Magnaudet (2008). When the Reynolds number is increased from sufficiently low values, the flow undergoes several successive bifurcations. A first steady bifurcation occurs for $R e \simeq 115$ : it breaks the axisymmetry but preserves the time invariance, leading to a three-dimensional steady state with a reflectional symmetry. A Hopf bifurcation then occurs for $R e \simeq 121$ : it breaks the remaining reflectional symmetry and the time invariance, leading to a fully three-dimensional periodic state. A third bifurcation finally occurs for $R e \simeq 140$, where the flow remains unsteady, but restores a reflectional symmetry normal to that preserved by the first bifurcation. Natarajan \& Acrivos (1993) have carried out a global stability analysis of the axisymmetric disk flow in the same range of Reynolds number. These authors have shown that the axisymmetric state is successively destabilized by a stationary mode of azimuthal wavenumber $m=1$ at $R e=116.5$ and by an oscillating mode of same azimuthal wavenumber at $R e=126.5$. One should note that the DNS calculations and the stability analysis provide consistent results concerning the first steady bifurcation, but that the critical Reynolds numbers corresponding to the onset of unsteadiness predicted by both approaches do not match. This could have been expected, though, as the analysis of Natarajan \& Acrivos (1993) considered only small disturbances superimposed on the axisymmetric base flow, and not on the three-dimensional state issuing from the first $m=1$ bifurcation.

In this study, since the two critical Reynolds numbers associated to the destabilization of the axisymmetric base flow are close to one another, we analytically compute the dynamics in the three-dimensional slow manifold supported by the stationary $m=1$ mode and the two oscillating $m= \pm 1$ modes identified by Natarajan \& Acrivos (1993). Owing to the specific symmetries of the flow, i.e. invariance under time translation and $O(2)$ symmetry, if we retain only the lowest order nonlinear terms, the normal form describing this interaction should read

$$
\begin{aligned}
\dot{A} & =\lambda_{A} A-\mu_{A} A|A|^{2}-v_{A} A\left|B^{+}\right|^{2}-v_{A}{ }^{*} A\left|B^{-}\right|^{2}-\chi_{A} B^{+} B^{-*} A^{*}, \\
\dot{B}^{+} & =\lambda_{B} B^{+}-\mu_{B} B^{+}\left|B^{+}\right|^{2}-v_{B} B^{+}\left|B^{-}\right|^{2}-\eta_{B} B^{+}|A|^{2}-\chi_{B} B^{-} A^{2}, \\
\dot{B}^{-} & =\lambda_{B} B^{-}-\mu_{B} B^{-}\left|B^{-}\right|^{2}-v_{B} B^{-}\left|B^{+}\right|^{2}-\eta_{B} B^{-}|A|^{2}-\chi_{B} B^{+} A^{* 2},
\end{aligned}
$$

where $A$ is the complex amplitude of the stationary mode, $B^{ \pm}$are the amplitudes of the two counter-rotating oscillating modes and the superscript $*$ stands for the complex conjugate. The normal form (1.2) is therefore generic as being the leadingorder system of polynomial differential equations that remains invariant under a translation of $t_{0}$ in time $\left(t \rightarrow t+t_{0}\right)$, a rotation of angle $\theta_{0}\left(\theta \rightarrow \theta+\theta_{0}\right)$ and reflection $(\theta \rightarrow-\theta)$. This imposes invariance of (1.2) under phase transformations

$$
\begin{aligned}
& \left(A, B^{+}, B^{-}\right) \longrightarrow\left(A, B^{+} \mathrm{e}^{\mathrm{i} \psi}, B^{-} \mathrm{e}^{\mathrm{i} \psi}\right) \quad\left(t \rightarrow t+t_{0}\right), \\
& \left(A, B^{+}, B^{-}\right) \longrightarrow\left(A \mathrm{e}^{\mathrm{i} \varphi}, B^{+} \mathrm{e}^{\mathrm{i} \varphi}, B^{-} \mathrm{e}^{-\mathrm{i} \varphi}\right) \quad\left(\theta \rightarrow \theta+\theta_{0}\right), \\
& \left(A, B^{+}, B^{-}\right) \longrightarrow\left(A^{*}, B^{-}, B^{+}\right) \quad(\theta \rightarrow-\theta),
\end{aligned}
$$

that may be easily verified (see Golubitsky, Stewart \& Schaeffer 1988, for details). It may also be verified that the normal form (1.2) is exhaustive, i.e. no other term of same polynomial order can be added to any of (1.2) without breaking the phase invariance.

In their study, Fabre et al. (2008) have deduced, from symmetry breaking considerations, a normal form of structure similar to (1.2) and estimated all coefficients by a trial and error procedure based on their DNS calculations. However, for the present codimension two bifurcation, the determination of the exact normal form (1.2) 
requires to compute 15 real coefficients. As will be discussed in $\S 4$, the sequence of bifurcations depends on the sign of only two coefficients whereas the precise threshold values depend on four coefficients. Consequently, an infinite number of coefficient set corresponds to the same bifurcation diagram, and the procedure followed by Fabre et al. (2008) does not result in a unique set of coefficients. One could imagine to estimate all 15 coefficients using transient dynamics computed by DNS. However, such a procedure, although it has been used successfully for codimension one bifurcations (Provansal et al. 1987), is a formidable task no one has ever attempted for multiple codimension problems. In the present study, we carry out a thoroughly analytical asymptotic expansion of the flow field based on the global modes destabilizing the axisymmetric wake. The normal form (1.2) is then rigorously derived at the third order of a standard weakly nonlinear analysis as the result of a Fredholm alternative applied to resonant terms. All coefficients of the normal form are analytically computed as the scalar product between an adjoint global mode and a resonant forcing terms determined by the first- and second-order solutions. These coefficients, which describe the nonlinear interactions between modes, are of particular interest since they determine the effective bifurcation sequence. Though, we would like to emphasize here that performing the analytical derivation of this normal form provides additional insight into the flow physics. The base flow modification and the harmonic generation are indeed exactly computed as part of the second-order solution. As will be shown in $\S 4$, this is essential to discuss some realistic features of the bifurcated flow. Moreover, each coefficient of (1.2) is shown to result from several nonlinear interactions between modes, that may be investigated separately. This study completes that of Fabre et al. (2008) by shedding new light on the physical origin of the coupling terms between modes, in particular we shall show that all interactions take place only in the recirculating bubble, which can therefore be viewed as the effective wavemaker. The resulting dynamics is then analysed and compared to that observed in the DNS computations. It turns out that our analytical study predicts with a remarkable precision the bifurcation diagram observed in Fabre et al. (2008), as well as the associated flow topologies.

The paper is organized as follows. The problem formulation is presented in $\S 2$. The global linear stability analysis is presented in $\S 3$, where we compute the leading modes and their associated adjoint modes that are required to compute the coefficients of the normal form. In $\S 4$, we carry out the standard weakly nonlinear analysis: the values of all coefficients of the normal form (1.3) are given and the associated sequence of bifurcations is discussed and compared to that observed in the DNS calculations of Fabre et al. (2008). The sensitivity of this sequence of bifurcations to experimental imperfections is discussed in $\S 5$.

\section{Flow configuration and methodology}

In the following, all quantities are made non-dimensional using $D$ and $U_{\infty}$. Standard cylindrical coordinates $r, \theta$ and $z$ with origin taken at the centre of the disk are used. The state vector $\boldsymbol{q}$ stands for the flow field $(\boldsymbol{u}, p)^{T}$, where $T$ designates the transpose, $\boldsymbol{u}=(u, v, w)^{T}$ is the fluid velocity where $u, v$ and $w$ are the radial, azimuthal and axial components and $p$ is the pressure. The fluid motion is governed by the incompressible Navier-Stokes equations

$$
\nabla \cdot \boldsymbol{u}=0, \quad \partial_{t} \boldsymbol{u}+\nabla \boldsymbol{u} \cdot \boldsymbol{u}+\nabla p-\frac{1}{R e} \nabla^{2} \boldsymbol{u}=0,
$$




$\begin{array}{ccccccc} & z_{-\infty} & z_{\infty} & r_{\infty} & n_{t} & \text { DoF } & \text { DoF } \\ M_{1} & -100 & 200 & 25 & 397992 & 1802527 & 2603136 \\ M_{2} & -100 & 150 & 25 & 362672 & 1642617 & 2372198 \\ M_{3} & -70 & 200 & 25 & 394173 & 1784759 & 2577497 \\ M_{4} & -100 & 200 & 20 & 379576 & 1720445 & 2484574 \\ M_{5} & -100 & 200 & 25 & 290609 & 1316891 & 1901769\end{array}$

TABLE 1. Properties of the meshes as a function of parameters $z_{-\infty}, z_{\infty}$ and $r_{\infty}$, corresponding to the inlet, outlet and lateral boundaries. Here $n_{t}$ is the number of triangles, $D o F_{0}$ is the number of degrees of freedom for a state vector $(u, w, p)^{T}$ used in the base flow calculations and $D o F_{m}$ is the number of degrees of freedom for a state vector $(u, v, w, p)^{T}$ used in the stability analysis. Meshes $M_{1}$ and $M_{2}$ have the same vertex densities but with a different location of the outlet boundary. In the same way, $M_{1}$ and $M_{3}$ differ by the location of the inlet boundary, while $M_{1}$ and $M_{4}$ differ by the location of the lateral boundary. $M_{1}$ and $M_{5}$ have the same spatial extent but $M_{5}$ is built with lower vertex densities.

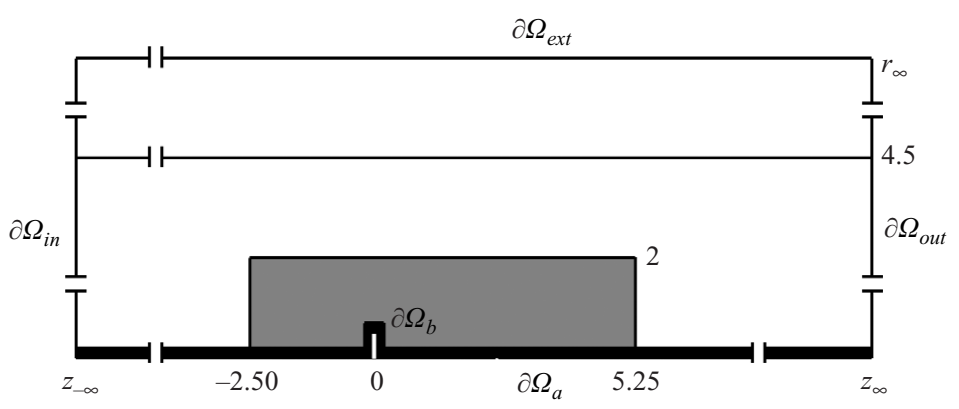

FIGURE 1. Schematic of the mesh structure: $z_{-\infty}, z_{\infty}$ and $r_{\infty}$ are, respectively, the location of the inlet, outlet and lateral boundaries. The inner solid lines delimit regions characterized by different vertex densities. The black shaded area corresponds to the region of highest vertex density and the grey shaded area is the near-wake region $\Omega_{i n}$ used for the normalization of the global modes.

with $R e=U_{\infty} D / v$, as previously introduced. The computational domain is shown in figure 1. The disk is located on the axis of an enclosing cylinder of radius $r=r_{\infty}$. The boundaries $\partial \Omega_{a}$ and $\partial \Omega_{e x t}$ represent the revolution axis of the disk and the boundary of the enclosing cylinder, respectively. The inlet $\partial \Omega_{\text {in }}$ and outlet $\partial \Omega_{\text {out }}$ are located respectively at $z=z_{-\infty}$ and $z=z_{\infty}$ (see table 1 for numerical values). We use the inlet conditions $\boldsymbol{u}=(0,0,1)^{T}$ on $\partial \Omega_{i n}$, no-slip conditions $\boldsymbol{u}=\mathbf{0}$ on the body wall $\partial \Omega_{b}$ and no-stress conditions $\left(-p \boldsymbol{I}+R e^{-1} \nabla \boldsymbol{u}\right) \cdot \boldsymbol{n}=\mathbf{0}$ on the outlet $\partial \Omega_{\text {out }}$. On the external boundary $\partial \Omega_{\text {ext }}$, we impose a free slip boundary condition $u=v=\partial_{r} w=0$, so that the body surface $\partial \Omega_{b}$ is the only source of vorticity, as would be the case without this artificial boundary. Note that for computational reasons, the width of the disk $L$ cannot be chosen strictly equal to zero, so that we use a very small width corresponding to an aspect ratio $L / D=10^{-3}$.

The numerical approach is based on a finite element method. A given equation is first multiplied by $r$ to avoid the singularity on the $r=0$ axis. Its associated variational formulation is then derived, and spatially discretized using a mesh composed of triangular elements. The FreeFem ++ software (http://www.freefem.org) is used to generate the triangulation with the Delaunay-Voronoi algorithm. The mesh refinement is controlled by the vertex densities on both external and internal 


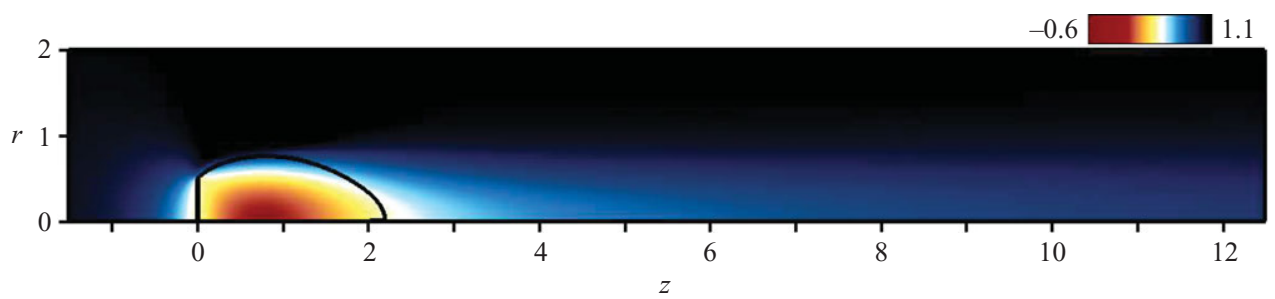

FiguRE 2. Steady axisymmetric base flow at the threshold of the first instability $(R e=116.9)$. The solid line in the flow indicates the separatrix of the recirculation zone.

boundaries. Regions where the mesh density varies are depicted in figure 1 . To avoid any computational difficulty, a zone of width 0.05 and high vertex density ( 250 vertex per unit length) is defined at the axis $r=0$ and around the disk, corresponding to the black shaded area in figure 1 . The unknown velocity and pressure fields $(\boldsymbol{u}, p)^{T}$ are spatially discretized using a basis of Taylor-Hood elements $(P 2$ elements for velocities and $P 1$ elements for pressure). The sparse matrices resulting from the projection of the variational formulations onto the basis of finite elements are built with the FreeFem ++ software. The matrix inverses are computed using the UMFPACK library, which consists in a sparse direct LU solver (Davis \& Duff 1997; Davis 2004). Five different meshes, denoted $M_{1}-M_{5}$ have been used to assess convergence in numerical results. These meshes exhibit various spatial extents and vertex densities, detailed in table 1 . The corresponding number of degrees of freedom for an axisymmetric and a three-dimensional state vector, such as those used in the base flow calculations and the stability analysis, respectively, are also provided. In the following, we will focus on the finest mesh $M_{1}$ to present all results. A comparison of the results obtained with the meshes $M_{1}-M_{5}$ is given in Appendix A.

\section{Linear analysis}

The aerodynamic flow field $\boldsymbol{q}=(\boldsymbol{u}, p)^{T}$ is decomposed into an axisymmetric steady base flow $\boldsymbol{q}^{0}=\left(u^{0}, 0, w^{0}, p^{0}\right)^{T}$ and a three-dimensional perturbation $\boldsymbol{q}^{\mathbf{1}}=$ $\epsilon\left(u^{1}, v^{1}, w^{1}, p^{1}\right)^{T}$ of amplitude $\epsilon$ assumed, in this section, infinitesimal.

\subsection{Base flow calculations}

The base flow $\boldsymbol{q}^{0}$ is sought as a steady axisymmetric solution of the Navier-Stokes equations:

$$
\nabla \cdot \boldsymbol{u}^{\mathbf{0}}=0, \quad \nabla \boldsymbol{u}^{\mathbf{0}} \cdot \boldsymbol{u}^{\mathbf{0}}+\nabla p^{0}-\frac{1}{R e} \nabla^{2} \boldsymbol{u}^{\mathbf{0}}=\mathbf{0} .
$$

On the axis $\partial \Omega_{a}$, the condition given by mass and momentum conservation as $r \rightarrow 0$ for axisymmetric solutions is $u^{0}=\partial_{r} w^{0}=0$. An approximate guess solution satisfying the required boundary conditions is first obtained by time marching the axisymmetric equations (2.1). The solution $\boldsymbol{q}^{\mathbf{0}}$ of the steady nonlinear equations (3.1) is then obtained using an iterative Newton method involving the resolution of simple linear problems, as described in Barkley, Gomes \& Henderson (2002) and Sipp \& Lebedev (2007). In the present study, the iterative process is carried out until the $\mathscr{L}^{2}$-norm of the residual of the governing equations for $\boldsymbol{q}^{0}$ becomes smaller than $10^{-12}$. Figure 2 shows contours of axial velocity $w^{0}$ of the base flow for $R e=116.9$. We observe a recirculation region of length $\simeq 2.1$ diameters, developing in the lee of the disk, with 
negative values of the axial velocity close to the axis reaching $60 \%$ of the free-stream velocity.

\subsection{Global eigenmode analysis}

At leading order in $\epsilon, \boldsymbol{q}^{\mathbf{1}}=\left(\boldsymbol{u}^{\mathbf{1}}, p^{1}\right)^{T}$ is a solution of the unsteady equations linearized about $\boldsymbol{q}^{\mathbf{0}}$ that read

$$
\mathscr{B} \partial_{t} \boldsymbol{q}^{\mathbf{1}}+\mathscr{A} \boldsymbol{q}^{\mathbf{1}}=\mathbf{0},
$$

where $\mathscr{A}$ and $\mathscr{B}$ are the linear operators defined by

$$
\mathscr{A}=\left(\begin{array}{cc}
\mathscr{C}\left(\cdot, \boldsymbol{u}^{\mathbf{0}}\right)-\frac{1}{R e} \nabla^{2} & \nabla \\
\nabla^{T} & 0
\end{array}\right), \quad \mathscr{B}=\left(\begin{array}{cc}
\mathscr{I} & 0 \\
0 & 0
\end{array}\right),
$$

and $\mathscr{C}(\boldsymbol{a}, \boldsymbol{b})$ is the advection operator $\nabla \boldsymbol{a} \cdot \boldsymbol{b}+\nabla \boldsymbol{b} \cdot \boldsymbol{a}$. Note that the operator $\mathscr{C}$ is symmetric, i.e. $\mathscr{C}(\boldsymbol{a}, \boldsymbol{b})=\mathscr{C}(\boldsymbol{b}, \boldsymbol{a})$. Since the base flow is axisymmetric, eigenmodes take the form of normal modes

$$
\boldsymbol{q}^{\mathbf{1}}=\hat{\boldsymbol{q}}^{\mathbf{1}}(r, z) \mathrm{e}^{(\sigma+\mathrm{i} \omega) t+\mathrm{i} m \theta}+\text { c.c. },
$$

where $\boldsymbol{q}^{1}=\left(\hat{u}^{1}, \hat{v}^{1}, \hat{w}^{1}, \hat{p}^{1}\right)^{T}$ is the so-called global mode for which both the crossstream and streamwise directions are eigendirections. The azimuthal wavenumber of the global mode is $m$, its growth rate and pulsation are $\sigma$ and $\omega$, respectively. Substitution of the development (3.4) in (3.2) leads to a generalized eigenvalue problem for $\sigma+\mathrm{i} \omega$ and $\hat{\boldsymbol{q}}^{\mathbf{1}}$ that reads

$$
(\sigma+\mathrm{i} \omega) \mathscr{B} \hat{\boldsymbol{q}}^{\mathbf{1}}+\mathscr{A}_{m} \hat{\boldsymbol{q}}^{\mathbf{1}}=\mathbf{0},
$$

where $\mathscr{A}_{m}$ is the linear operator

$$
\mathscr{A}_{m}=\left(\begin{array}{cc}
\mathscr{C}_{m, 0}\left(\cdot, \boldsymbol{u}^{\mathbf{0}}\right)-\frac{1}{R e} \nabla_{\boldsymbol{m}}{ }^{2} & \nabla_{\boldsymbol{m}} \\
\nabla_{\boldsymbol{m}}{ }^{T} & 0
\end{array}\right) .
$$

In (3.6), $\nabla_{m}$ is the gradient operator relative to the azimuthal wavenumber $m$, and $\mathscr{C}_{m, n}(\hat{\boldsymbol{a}}, \hat{\boldsymbol{b}})$ is the symmetric advection operator for a pair of normal modes $\hat{\boldsymbol{a}}$ and $\hat{\boldsymbol{b}}$ of respective azimuthal wavenumbers $m$ and $n$, i.e. $\mathscr{C}_{m, n}(\hat{\boldsymbol{a}}, \hat{\boldsymbol{b}})=\nabla_{\boldsymbol{m}} \hat{\boldsymbol{a}} \cdot \hat{\boldsymbol{b}}+\nabla_{\boldsymbol{n}} \hat{\boldsymbol{b}} \cdot \hat{\boldsymbol{a}}$. Owing to the normal mode expansion (3.4), these operators are complex, since $\theta$ derivatives introduce product by $\mathrm{i} m$, and a complete expansion of these operators can be found in Appendix C. The global mode satisfies the boundary conditions

$$
\begin{aligned}
\hat{\boldsymbol{u}}^{\mathbf{1}}=\mathbf{0} & \text { on } \partial \Omega_{\text {in }} \cup \partial \Omega_{b} \text { (inlet and body), } \\
\left(-\hat{p}^{1} \boldsymbol{I}+\operatorname{Re}^{-1} \nabla_{\boldsymbol{m}} \hat{\boldsymbol{u}}^{\mathbf{1}}\right) \cdot \boldsymbol{n}=\mathbf{0} & \text { on } \partial \Omega_{\text {out }} \text { (outlet) } \\
\hat{u}^{1}=\partial_{r} \hat{v}^{1}=\partial_{r} \hat{w}^{1}=0 & \text { on } \partial \Omega_{\text {ext }} \text { (external boundary). }
\end{aligned}
$$

The conditions at the axis $\partial \Omega_{a}$ depend on the azimuthal wavenumber $m: \hat{u}^{1}=\partial_{r} \hat{w}^{1}=$

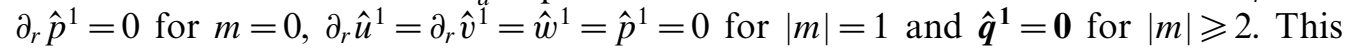
eigenvalue problem is solved using an Arnoldi method based on a shift-invert strategy, as in Ehrenstein \& Gallaire (2005). Owing to the symmetries of the problem, one should note that system (3.5) is invariant under the $\left(\hat{u}^{1}, \hat{v}^{1}, \hat{w}^{1}, \hat{p}^{1}, m\right) \rightarrow$ $\left(\hat{u}^{1},-\hat{v}^{1}, \hat{w}^{1}, \hat{p}^{1},-m\right)$ transformation, so that we investigate only the case $m \geqslant 0$ in this section. Moreover, if $\left(\hat{\boldsymbol{q}}^{\mathbf{1}}, \sigma+\mathrm{i} \omega\right)$ is solution of problem (3.5), then $\left(\hat{\boldsymbol{q}}^{\mathbf{1}^{*}}, \sigma-\mathrm{i} \omega\right)$ is also a solution, i.e. eigenvalues are complex conjugates, so that all spectra in the $(\sigma, \omega)$ plane are symmetric with respect to the real axis.

In the following, $\langle$,$\rangle is the inner product defined by \langle\hat{\boldsymbol{a}}, \hat{\boldsymbol{b}}\rangle=\int_{\Omega} \hat{\boldsymbol{a}}^{*} \cdot \hat{\boldsymbol{b}} r \mathrm{~d} r \mathrm{~d} z$ where $\hat{\boldsymbol{a}}$ and $\hat{\boldsymbol{b}}$ belong to $\mathbb{C}^{n}$ and $\cdot$ refers to the canonical hermitian scalar product in $\mathbb{C}^{n}$. 
To normalize the global modes, we define arbitrarily a near-wake domain $\Omega_{i n}$ as $z \in[-2.5,5.25]$ and $r<2$, corresponding to the grey shaded area in figure 1. All global modes are normalized by imposing first the phase of the radial velocity to be zero at a particular location, namely $\hat{u}^{1}(0,1)$ must be real positive for $m= \pm 1$. The eigenmode energy is then normalized to unity in $\Omega_{i n}$ by imposing that $\left\langle\hat{\boldsymbol{q}}^{\mathbf{1}}, \delta_{i n} \mathscr{B} \hat{\boldsymbol{q}}^{\mathbf{1}}\right\rangle=1$, where $\delta_{i n}$ is the function defined as $\delta_{i n}(r, z)=1$ if $(r, z) \in \Omega_{\text {in }}$ and 0 otherwise. These normalization choices have no effect on the dynamics but ease the comparison between results when the convergence tests are carried out.

For a given global mode $\hat{\boldsymbol{q}}^{\mathbf{1}}$, we also compute its adjoint global mode $\hat{\boldsymbol{q}}^{\mathbf{1}}$ that verifies the adjoint eigenvalue problem

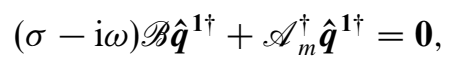

where $\mathscr{A}_{m}^{\dagger}$ is the adjoint of operator $\mathscr{A}_{m}$, obtained by integrating by parts system (3.5) (see Schmid \& Henningson 2001, for details)

$$
\mathscr{A}_{m}^{\dagger}=\left(\begin{array}{cc}
\mathscr{C}_{m, 0}^{\dagger}\left(\cdot, \boldsymbol{u}^{\mathbf{0}}\right)-\frac{1}{R e} \nabla_{\boldsymbol{m}}{ }^{2} & -\nabla_{\boldsymbol{m}} \\
\nabla_{\boldsymbol{m}}{ }^{T} & 0
\end{array}\right) .
$$

$\mathscr{C}_{m, n}^{\dagger}(\hat{\boldsymbol{a}}, \hat{\boldsymbol{b}})=\nabla_{n} \hat{\boldsymbol{b}}^{T} \cdot \hat{\boldsymbol{a}}-\nabla_{m} \hat{\boldsymbol{a}} \cdot \hat{\boldsymbol{b}}$ is the adjoint advection operator. It is worthwhile noting that $\mathscr{C}_{m, n}^{\dagger}$ is not symmetric. We would like to emphasize that the terms describing the convection of disturbances by the base flow, namely $\nabla_{m} \hat{\boldsymbol{u}}^{\mathbf{1}} \cdot \boldsymbol{u}^{\mathbf{0}}$ and $-\nabla_{m} \hat{\boldsymbol{u}}^{\mathbf{1} \dagger} \cdot \boldsymbol{u}^{\mathbf{0}}$ have opposite signs between the direct and adjoint advection operators. As a result, disturbances are convected downstream for $\mathscr{A}_{m}$ and upstream for $\mathscr{A}_{m}^{\dagger}$, inducing a spatial separation of direct and adjoint modes (Chomaz, Huerre \& Redekopp 1990). This convective non-normality is specific to open flows (Chomaz 2005).

The adjoint boundary conditions are defined so that all boundary terms arising from the integration by parts are nil. We obtain

$$
\begin{aligned}
& \hat{\boldsymbol{u}}^{1 \dagger}=\mathbf{0} \text { on } \partial \Omega_{i n} \cup \partial \Omega_{b}, \\
& \left(\boldsymbol{u}^{\mathbf{0}} \cdot \boldsymbol{n}\right) \hat{\boldsymbol{u}}^{1 \dagger}+\left(\hat{p}^{1 \dagger} \boldsymbol{I}+R e^{-1} \nabla_{\boldsymbol{m}} \hat{\boldsymbol{u}}^{1 \dagger}\right) \cdot \boldsymbol{n}=\mathbf{0} \text { on } \partial \Omega_{\text {out }}, \\
& \hat{u}^{1 \dagger}=\partial_{r} \hat{v}^{1 \dagger}=\partial_{r} \hat{w}^{1 \dagger}=0 \text { on } \partial \Omega_{e x t} .
\end{aligned}
$$

The condition on the axis $\partial \Omega_{a}$ is identical to that applied to the direct global mode. This eigenproblem is solved via the same Arnoldi method, and adjoint global modes are normalized so that $\left\langle\hat{\boldsymbol{q}}^{\mathbf{1} \dagger}, \mathscr{B} \hat{\boldsymbol{q}}^{\mathbf{1}}\right\rangle=1$. Since the adjoint problem (3.8) has been formulated for continuous operators with associated adjoint boundary conditions, the spatial discretizations of problems (3.5) and (3.8) are not hermitian to one another, because operator $\mathscr{B}$ does not correspond to the scalar product in cylindrical coordinates. Consequently, we check a posteriori that both problems have identical spectra, and that the bi-orthogonality relation is satisfied for the 10 leading global modes, as will be detailed in the following.

In the linear framework, we compute the leading global modes and their adjoint global modes, that will be used in $\S 4$ to compute the coefficients of the normal form (1.2). The threshold Reynolds numbers and characteristics of the destabilizing global modes agree with the results of Natarajan \& Acrivos (1993): the first instability is steady (i.e. $\omega_{A}=0$ ) and occurs at $R e_{c}^{A}=116.9$ for $m=1$ and the second instability occurs at $R e_{c}^{B}=125.3$ for an $m=1$ oscillating mode of frequency $\omega_{B}=0.760$, corresponding to a Strouhal number $S t_{B}=\omega_{B} D /\left(2 \pi U_{\infty}\right)$ of 0.121. Figure 3(a) shows the eigenvalue spectra at the first critical Reynolds number $R e_{c}^{A} \cdot m=1$ modes are shown as diamonds in the upper half-plane whereas $m=0$ and $m=2$ modes are 

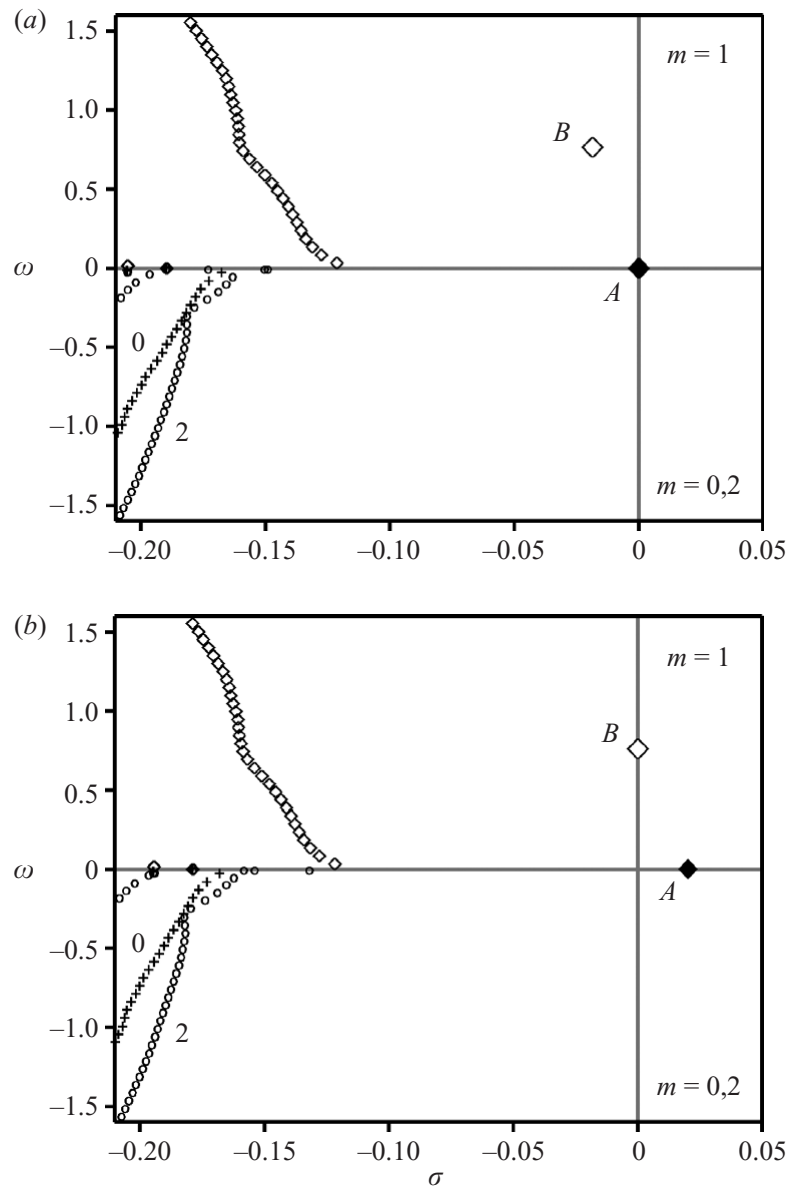

FiguRE 3. $m=0,1,2$ eigenvalue spectra in the $(\sigma, \omega)$ plane for the wake of a disk. $(a)$ Threshold of the first instability at $\operatorname{Re}_{c}^{A}=116.9$. (b) Threshold of the second instability at $R e_{c}^{B}=125.3$. All spectra are symmetric with respect to the real axis. The upper half-plane shows $m=1$ disturbances as diamonds, and the lower half-plane shows $m=0,2$ disturbances as + and $o$ symbols, respectively. The large diamonds labelled $A$ and $B$ correspond respectively to the steady and oscillating destabilizing $m=1$ modes.

shown respectively as + and $o$ symbols in the lower half-plane. The marginally unstable eigenvalue labelled $A$ vanishes at threshold and corresponds to the large closed diamond. In the following, the eigenvector associated to this steady global mode is denoted $\hat{\boldsymbol{q}}_{A}^{\mathbf{1}}$. Figure $4(a)$ shows the spatial structure of the axial velocity $\hat{w}_{A}^{1}$ : one observes a low-speed region extending far downstream. Since the azimuthal wavenumber of this global mode is $m=1$, the axial velocity perturbation is opposite on the other side of the revolution axis, which induces an increase of the streamwise velocity of the total flow. The stationary global mode therefore mainly induces an off-axis displacement of the wake. The associated adjoint global mode $\hat{\boldsymbol{q}}_{A}^{\mathbf{1} \dagger}$ is presented in figure $4(b)$. It is dominated by high magnitudes of adjoint axial velocity $\hat{w}_{A}^{1 \dagger}$ within the recirculating area, the maximum value being reached close to the separating streamline, but it is also intense far upstream of the disk. As mentioned previously, the downstream localization of the global mode and the upstream localization of the adjoint global mode result from the convective non-normality of the linearized 

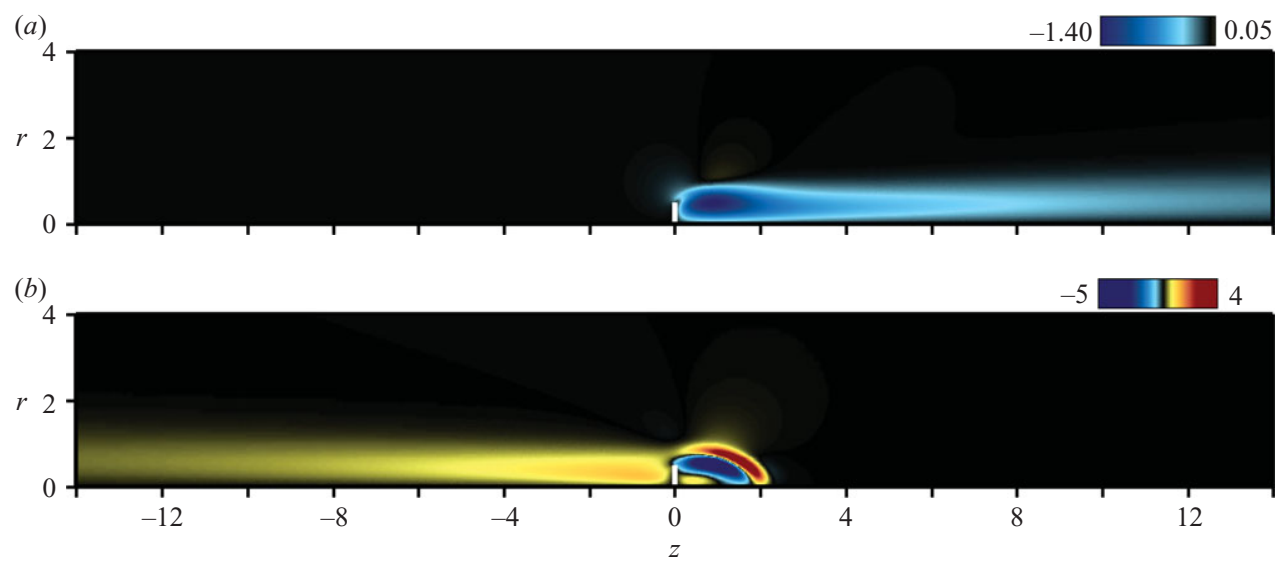

FIGURE 4. Steady global mode and adjoint global mode at the threshold of the first instability, $R e_{c}^{A}=116.9$. (a) Spatial distribution of axial velocity $\hat{w}_{A}^{1}$ for the global mode. (b) Spatial distribution of axial velocity $\hat{w}_{A}^{1 \dagger}$ for the adjoint global mode. The black hue corresponds to vanishing perturbations.
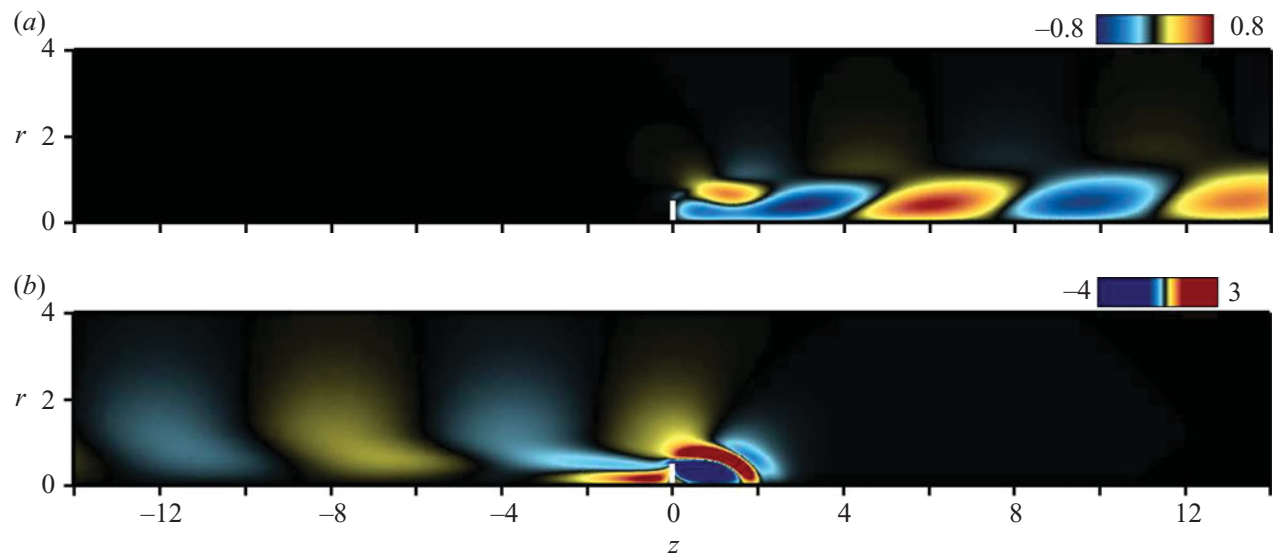

FIGURE 5. Same as figure (4) for the oscillating global mode at threshold. Only the real part is shown here.

Navier-Stokes operator (see Chomaz 2005). Figure 3(b) shows the eigenvalue spectra at the second instability threshold $R e_{c}^{B}=125.3$. The eigenvalue corresponding to the first destabilizing eigenmode $\hat{\boldsymbol{q}}_{A}^{\mathbf{1}}$ has moved to the unstable $(\sigma>0)$ half-plane. Moreover, the oscillating eigenvalue labelled $B$, corresponding to the large open diamond, which was in the stable domain $(\sigma<0)$ in figure $3(a)$, is now crossing the $\sigma=0$ axis. The marginally unstable eigenvalue $\mathrm{i} \omega_{B}$ is associated to the eigenvector denoted $\hat{\boldsymbol{q}}_{\boldsymbol{B}^{+}}^{\mathbf{1}}$, and $\hat{\boldsymbol{q}}_{\boldsymbol{B}^{-}}^{\mathbf{1}}$ stands for the symmetric eigenmode associated to the azimuthal wavenumber $m=-1$ for the same eigenvalue $\mathrm{i} \omega_{B}$. Since these modes are oscillating, their eigenvectors are complex, and figure 5(a) shows the spatial structure of the real part of $\hat{w}_{B^{+}}^{1}$. One observes positive and negative velocity perturbations alternating downstream of the disk, in a regular periodic way that allows to define a local spatial wavelength of about 7 diameters. The imaginary part of $\hat{w}_{B^{+}}^{1}$ (not shown here) displays a similar structure, but is approximately in spatial quadrature since its extrema are located where the real part vanishes. This global mode corresponds therefore to a 


\begin{tabular}{ccccccc}
\hline$\hat{\boldsymbol{q}}^{1}$ & $A$ & $B$ & $C$ & $D$ & $E$ & $F$ \\
$\gamma$ & 1 & $1.87 \times 10^{-7}$ & $6.31 \times 10^{-9}$ & $3.01 \times 10^{-7}$ & $1.71 \times 10^{-7}$ & $2.22 \times 10^{-9}$
\end{tabular}

TABLE 2. Value of the $\gamma$ parameter defined by (3.11) computed for the six leading eigenmodes of azimuthal wavenumber $m=1$ at the second instability threshold $\operatorname{Re}_{c}^{B}=125.3$. The eigenmodes labelled $A$ and $B$ are the two leading modes discussed in the present study, and the subsequent modes are labelled $C, D, E$ and $F$.

spiral perturbation in the lee of the disk, which rotates in time at the frequency $\omega_{B}$. One should note that the eigenmode for the $m=-1$ perturbation is similar, but has the opposite pitch and rotates in time in the opposite direction at the same frequency $\omega_{B}$. As for the stationary mode, the adjoint global mode $\hat{\boldsymbol{q}}_{\boldsymbol{B}^{+}}^{\mathbf{1}}$ shown in figure $5(b)$ is intense only in the recirculating area and a few diameters upstream of the disk, where it presents a weak oscillation, and it vanishes downstream of the disk. As a way to quantify the precision of the present computations, we present in table 2 the magnitude of the ratio

$$
\gamma=\frac{\left\langle\hat{\boldsymbol{q}}_{A}^{\mathbf{1}}, \mathscr{B} \hat{\boldsymbol{q}}^{\mathbf{1}}\right\rangle}{\left\langle\hat{\boldsymbol{q}}_{A}^{\mathbf{1}}, \mathscr{B} \hat{\boldsymbol{q}}_{A}^{\mathbf{1}}\right\rangle}
$$

for the six leading eigenmodes of azimuthal wavenumber $m=1$ at the second instability threshold $\operatorname{Re}_{c}^{B}=125.3$. It can be seen that the bi-orthogonality condition is respected with excellent precision, since $\gamma$ is less than $10^{-7}$ if $\hat{\boldsymbol{q}}^{\mathbf{1}} \neq \hat{\boldsymbol{q}}_{\boldsymbol{A}}^{\mathbf{1}}$. As a consequence, we can conclude that our numerical procedure accurately estimates the direct and adjoint global modes.

\section{Weakly nonlinear analysis}

\subsection{Presentation}

Since the critical Reynolds numbers for both destabilizing modes are close to one another, we implement here an asymptotic expansion where these three modes have the same order of magnitude. The Reynolds number $R e$ is assumed to vary in a range close to the mean critical Reynolds number $R e_{c}=\left(R e_{c}^{A}+R e_{c}^{B}\right) / 2=121.1$. The departure from criticality is assumed to be of order $\epsilon^{2}$. Therefore, we introduce the order unity parameter $\delta$, such that

$$
\frac{1}{R e}=\frac{1}{R e_{c}}-\epsilon^{2} \delta
$$

The threshold Reynolds numbers $R e_{c}^{A}$ and $R e_{c}^{B}$ are then rescaled into the criticality parameters $\delta^{A}$ and $\delta^{B}$, so that

$$
\frac{1}{R e_{c}^{A}}=\frac{1}{R e_{c}}-\epsilon^{2} \delta^{A}, \quad \frac{1}{R e_{c}^{B}}=\frac{1}{R e_{c}}-\epsilon^{2} \delta^{B} .
$$

In practice, $\epsilon$ is chosen equal to $10^{-1}$, so that $\delta^{A}=-2.97 \times 10^{-2}$ and $\delta^{B}=2.77 \times 10^{-2}$. However, the results, and in particular the final bifurcation diagram, are mainly insensitive to the precise choice of $R e_{c}$ and of $\epsilon$, as shown in appendix B. As mentioned in the introduction, the weakly nonlinear analysis requires the introduction of multiple time scales with a fast time scale $t$ and a slow time scale $t_{1}=\epsilon^{2} t$. The 
$\partial_{t}$ term in (2.1) is therefore transformed into $\partial_{t}+\epsilon^{2} \partial_{t_{1}}$. Note that the growth rates $\sigma_{A}$ and $\sigma_{B}$ are non-zero at $\operatorname{Re}_{c}$, since the stationary mode $\hat{\boldsymbol{q}}_{A}^{1}$ is slightly unstable and the oscillating modes $\hat{\boldsymbol{q}}_{\boldsymbol{B}^{ \pm}}^{1}$ are slightly stable. However, with the present scaling assumption, the growth rates $\sigma_{A}$ and $\sigma_{B}$ of these modes differ from zero only at order $\epsilon^{2}$. We define $\omega_{0}$ as the frequency of modes $\hat{\boldsymbol{q}}_{\boldsymbol{B}^{ \pm}}^{1}$ at the mean critical Reynolds number $R e_{c}$, i.e. $\omega_{0}=\omega_{B}\left(R e_{c}\right)=0.764$, so that at $R e_{c}$,

$$
\begin{aligned}
\sigma_{A} & =\epsilon^{2} \tilde{\sigma}_{A}, \\
\sigma_{B}+\mathrm{i} \omega_{B} & =\mathrm{i} \omega_{0}+\epsilon^{2} \tilde{\sigma}_{B},
\end{aligned}
$$

where the second-order growth rates $\tilde{\sigma}_{A}$ and $\tilde{\sigma}_{B}$ are assumed to be of order unity. This second-order departure from neutrality is taken into account by replacing the leading-order operator $\mathscr{A}_{m}^{c}=\mathscr{A}_{m}\left(R \boldsymbol{e}_{c}\right)$ defined in (3.6), for which $\hat{\boldsymbol{q}}_{\boldsymbol{A}}^{1}$ and $\hat{\boldsymbol{q}}_{\boldsymbol{B}^{ \pm}}^{1}$ are not neutral, by the shifted operator $\widetilde{\mathscr{A}}_{m}^{c}=\mathscr{A}_{m}^{c}-\epsilon^{2} \mathscr{S}_{m}$, where $\mathscr{S}_{m}$ is the shift operator defined by

$$
\begin{aligned}
\mathscr{S}_{1} \hat{\boldsymbol{q}}_{\boldsymbol{A}}^{1} & =\tilde{\sigma}_{A} \hat{\boldsymbol{q}}_{A}^{1}, \\
\mathscr{S}_{1} \hat{\boldsymbol{q}}_{\boldsymbol{B}^{+}}^{1} & =\tilde{\sigma}_{B} \hat{\boldsymbol{q}}_{\boldsymbol{B}^{+}}, \\
\mathscr{S}_{-1} \hat{\boldsymbol{q}}_{B^{-}}^{1} & =\tilde{\sigma}_{B} \hat{\boldsymbol{q}}_{\boldsymbol{B}^{-}}, \\
\mathscr{S}_{ \pm 1} \hat{\boldsymbol{q}} & =\mathbf{0} \text { for the remaining } m= \pm 1 \text { modes, } \\
\mathscr{S}_{m} \hat{\boldsymbol{q}} & =\mathbf{0} \text { for all other } m .
\end{aligned}
$$

It is worthwhile emphasizing that $\widetilde{\mathscr{A}}_{m}^{c}$ has precisely the same spectra than $\mathscr{A}_{m}^{c}$ at the critical Reynolds number $\operatorname{Re}_{c}$, excepted that $\hat{\boldsymbol{q}}_{\boldsymbol{A}}^{1}$ and $\hat{\boldsymbol{q}}_{\boldsymbol{B}^{ \pm}}^{1}$ are now neutral. The shift operator is required only to apply the formalism of weakly nonlinear analyses to a rigourous multiple codimension bifurcation. However, this operator is only formal and in practice, we do not need to construct it explicitely, since its effect is simply to bring the growth rates $\sigma_{A}$ and $\sigma_{B}$ to zero at the same Reynolds number, without changing the eigenmodes shape. To the authors' knowledge, the use of such a method has not been reported yet, but it is somehow straightforward.

The flow field $\boldsymbol{q}$ is expanded as

$$
\boldsymbol{q}=\boldsymbol{q}^{\mathbf{0}}+\epsilon \boldsymbol{q}^{\mathbf{1}}+\epsilon^{2} \boldsymbol{q}^{\mathbf{2}}+\epsilon^{3} \boldsymbol{q}^{3}+\cdots
$$

and the governing equations (2.1) then give rise to a series of equations at successive orders of $\epsilon$.

\subsection{Orders 0 and 1}

At order $\epsilon^{0}$, the equations are the nonlinear equations (2.1) for the Reynolds number $R e_{c}$, i.e. $\boldsymbol{q}^{0}$ is the steady axisymmetric solution computed, as in $\S 3.1$, for $R e_{c}$. The equations at order $\epsilon^{1}$ are the linearized equations given by (3.2) at $R e_{c}$ :

$$
\mathscr{B} \partial_{t} \boldsymbol{q}^{\mathbf{1}}+\widetilde{\mathscr{A}}^{c} \boldsymbol{q}^{\mathbf{1}}=\mathbf{0},
$$

where $\widetilde{\mathscr{A}}^{c}$ is the shifted evolution operator acting in the real space, at the critical Reynolds number $R e_{c}$, obtained from $\widetilde{\mathscr{A}}_{m}^{c}$ by inverse Fourier transform in time and in the azimuthal direction. Equation (4.6) specifies that $\boldsymbol{q}^{\mathbf{1}}$ is a superposition of eigenmodes destabilizing the steady state $\boldsymbol{q}^{\mathbf{0}}$ :

$$
\boldsymbol{q}^{1}=A \hat{\boldsymbol{q}}_{A}^{1} \mathrm{e}^{\mathrm{i} \theta}+B^{+} \hat{\boldsymbol{q}}_{\boldsymbol{B}^{+}}^{1} \mathrm{e}^{\mathrm{i} \theta+\mathrm{i} \omega_{0} t}+B^{-} \hat{\boldsymbol{q}}_{\boldsymbol{B}^{-}}^{1} \mathrm{e}^{-\mathrm{i} \theta+\mathrm{i} \omega_{0} t}+\text { c.c. }
$$




\begin{tabular}{ccccccccccccc}
\hline & $A A^{*}$ & $B^{+} B^{+*}$ & $B^{-} B^{-*}$ & $A A$ & $B^{+} B^{+}$ & $B^{-} B^{-}$ & $B^{+} A$ & $B^{+} A^{*}$ & $B^{+} B^{-}$ & $B^{+} B^{-*}$ & $B^{-} A$ & $B^{-} A^{*}$ \\
$m$ & 0 & 0 & 0 & 2 & 2 & -2 & 2 & 0 & 0 & 2 & 0 & -2 \\
$\omega$ & 0 & 0 & 0 & 0 & $2 \omega_{0}$ & $2 \omega_{0}$ & $\omega_{0}$ & $\omega_{0}$ & $2 \omega_{0}$ & 0 & $\omega_{0}$ & $\omega_{0}$
\end{tabular}

TABLE 3. Order 2 nonlinear forcing terms gathered by their amplitude dependency, and corresponding azimuthal and temporal periodicity $(m, \omega)$. Nine terms have been omitted as they are complex conjugated of the ones presented here.

where $A$ is the complex amplitude of the steady mode $\hat{\boldsymbol{q}}_{A}^{1}$, and $B^{+}$and $B^{-}$are the complex amplitudes of the oscillating mode $\hat{\boldsymbol{q}}_{B^{+}}^{1}$ and $\hat{\boldsymbol{q}}_{B^{-}}^{1}$ respectively, $\left(A, B^{+}, B^{-}\right)$ being, at this stage, unknown functions of the slow time $t_{1}$.

\subsection{Order 2}

At order $\epsilon^{2}$ we obtain the linearized Navier-Stokes equations applied to $\boldsymbol{q}^{2}$ :

$$
\mathscr{B} \partial_{t} \boldsymbol{q}^{2}+\widetilde{\mathscr{A}}^{c} \boldsymbol{q}^{2}=\boldsymbol{F}^{2},
$$

forced by a term $\boldsymbol{F}^{2}$ depending only on zero and first-order solutions

$$
\boldsymbol{F}^{\mathbf{2}}=-\left(\delta \nabla^{2} \boldsymbol{u}^{\mathbf{0}}+\mathscr{C}\left(\boldsymbol{u}^{\mathbf{1}}, \boldsymbol{u}^{\mathbf{1}}\right), 0\right)^{T} .
$$

The first term $-\delta \nabla^{2} \boldsymbol{u}^{\mathbf{0}}$ in (4.9) is linear and arises from the Reynolds number variation, quantified by $\delta$ and acting here on the base flow. The other contribution $-\mathscr{C}\left(\boldsymbol{u}^{\mathbf{1}}, \boldsymbol{u}^{\mathbf{1}}\right)$ is nonlinear and is due to the transport of the first-order solution $\boldsymbol{q}^{\mathbf{1}}$ by itself. Since the first-order solution is made of six different contributions of respective amplitudes $A, A^{*}, B^{+}, B^{+*}, B^{-}$and $B^{-*}$, its self-transport generates 21 different nonlinear terms. Each of these terms, denoted $\hat{\boldsymbol{F}}_{i j}^{2} \mathrm{e}^{(\mathrm{i} m \theta+\mathrm{i} \omega t)}$ (the subscripts $i$ and $j$ stand for one of the six first-order amplitudes) exhibits a specific spatial periodicity $m$ and frequency $\omega$, gathered in table 3. These forcing terms are non-resonant, since associated to azimuthal wavenumbers different from $m= \pm 1$, so that the forced equation (4.8) can be inverted. The second-order solution $\overline{\boldsymbol{q}}^{2}$ is thus sought as the superposition of the response $\boldsymbol{q}_{\delta}^{2}$ to the viscous forcing term $-\delta \nabla^{2} \boldsymbol{u}^{\mathbf{0}}$ of (4.9), which describes the axisymmetric base flow modification when the Reynolds number is varied, and of the 21 responses $\hat{\boldsymbol{q}}_{i j}^{2}$ to each individual forcing terms $\hat{\boldsymbol{F}}_{i j}^{2}$, i.e.

$$
\begin{aligned}
& \mathbf{q}^{2}=\delta \hat{\boldsymbol{q}}_{\delta}^{2}+|A|^{2} \hat{\boldsymbol{q}}_{\boldsymbol{A} A^{*}}^{2}+\left|B^{+}\right|^{2} \hat{\boldsymbol{q}}_{\boldsymbol{B}^{+} \boldsymbol{B}^{+*}}^{2}+\left|B^{-}\right|^{2} \hat{\boldsymbol{q}}_{\boldsymbol{B}^{-} \boldsymbol{B}^{-*}}^{\mathbf{2}} \\
& +A^{2} \hat{\boldsymbol{q}}_{\boldsymbol{A} A}^{2} \mathrm{e}^{2 \mathrm{i} \theta}+B^{+} B^{-*} \hat{\boldsymbol{q}}_{\boldsymbol{B}^{+} \boldsymbol{B}^{-*}}^{2} \mathrm{e}^{2 \mathrm{i} \theta}+\text { c.c. }
\end{aligned}
$$

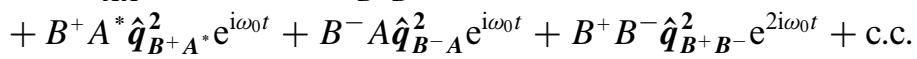

$$
\begin{aligned}
& +B^{+2} \hat{\boldsymbol{q}}_{\boldsymbol{B}^{+} \boldsymbol{B}^{+}}^{2} \mathrm{e}^{2 \mathrm{i} \theta+2 \mathrm{i} \omega_{0} t}+B^{-2} \hat{\boldsymbol{q}}_{\boldsymbol{B}^{-} \boldsymbol{B}^{-}}^{2} \mathrm{e}^{-2 \mathrm{i} \theta+2 \mathrm{i} \omega_{0} t}+\text { c.c. } \\
& +B^{+} A \hat{\boldsymbol{q}}_{\boldsymbol{B}^{+} A^{2}}^{2} \mathrm{e}^{2 \mathrm{i} \theta+\mathrm{i} \omega_{0} t}+B^{-} A^{*} \hat{\boldsymbol{q}}_{\boldsymbol{B}^{-} A^{*}}^{2} \mathrm{e}^{-2 \mathrm{i} \theta+\mathrm{i} \omega_{0} t}+\text { c.c.. }
\end{aligned}
$$

Each response $\hat{\boldsymbol{q}}_{i j}^{2}$ is solution of a linear problem

$$
\left(\mathrm{i} \omega \mathscr{B}+\tilde{\mathscr{A}}^{c}\right) \hat{\boldsymbol{q}}_{i j}^{2}=\hat{\boldsymbol{F}}_{i j}^{2},
$$

with $m$ and $\omega$ for each couple $(i, j)$ being collected from table 3 . As already mentioned, none of the combinations $(m, \omega)$ gathered in table 3 is an eigenvalue in figure 3 since none of them is $m= \pm 1$, so that $i \omega \mathscr{B}+\widetilde{\mathscr{A}}^{c}$ are always non-degenerate linear operators. The axial velocity components of some of the 21 forcing terms and of their associated responses are shown in figure 6 . The transport of the stationary mode of amplitude $A$ by itself generates the forcing term $\hat{\boldsymbol{F}}_{\boldsymbol{A} A}^{2}$ of azimuthal wavenumber $m=2$ and 


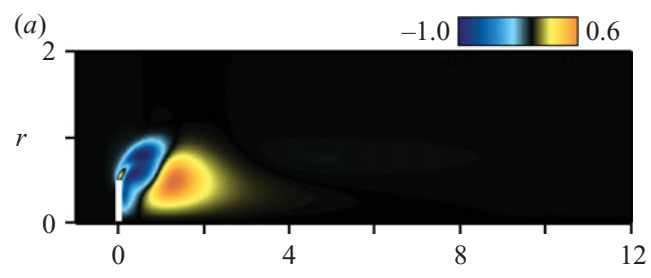

(b)
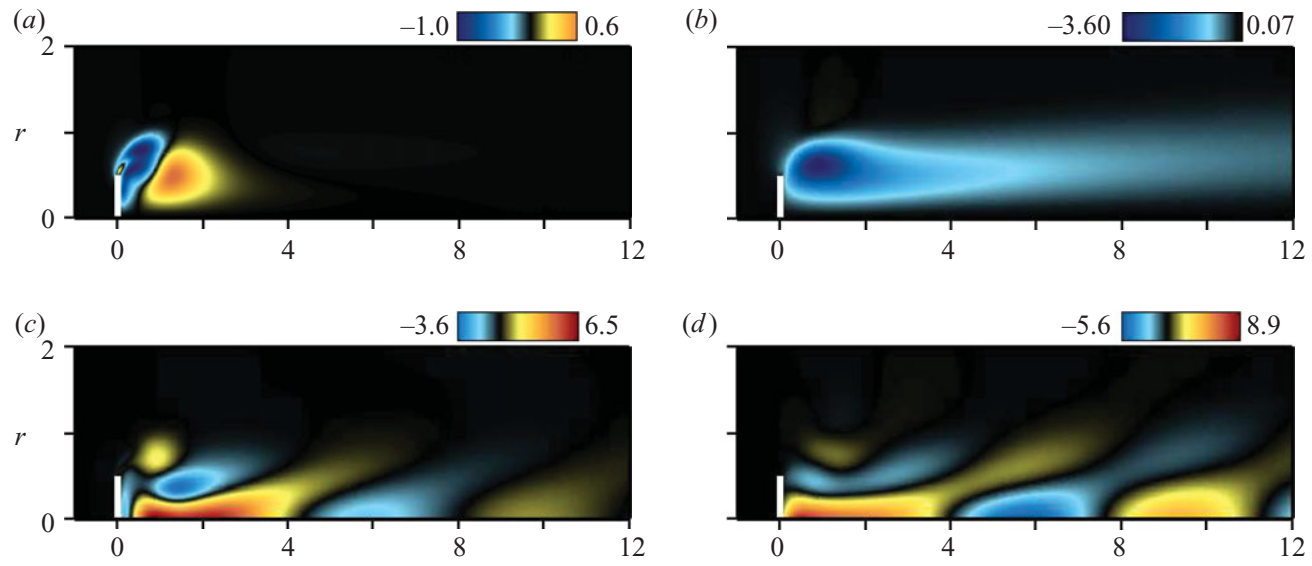

(d)
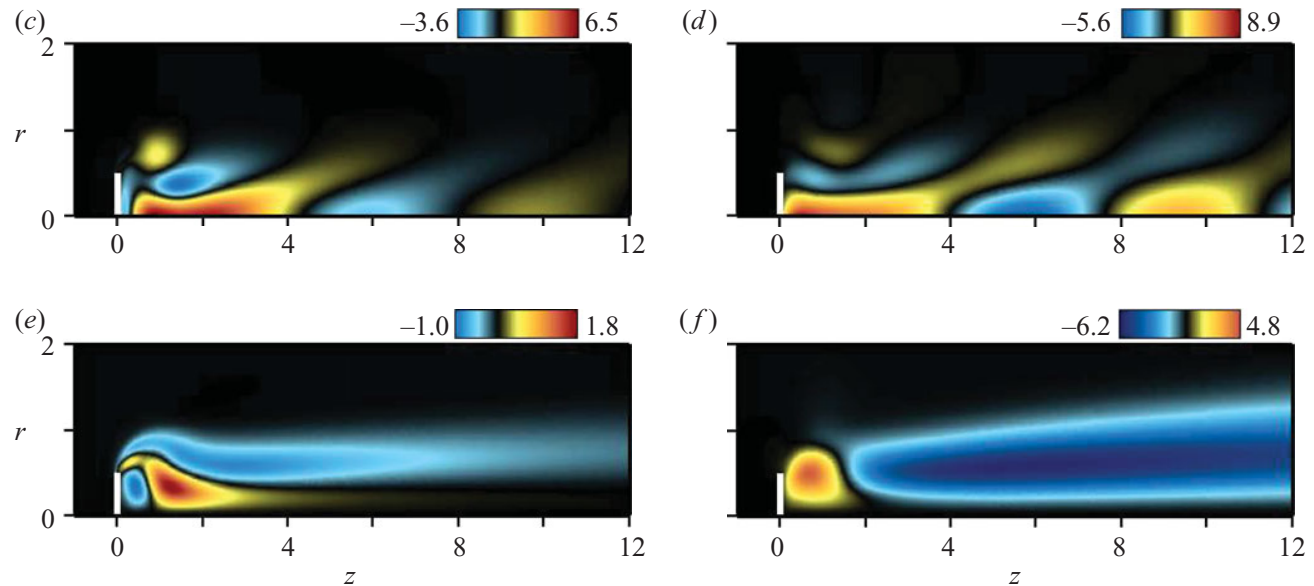

FIGURE 6. Second-order forcing terms and associated responses: representation of various flow fields appearing at order $\epsilon^{2}$ in the weakly nonlinear analysis. Figures on the left show the real part of the axial component of the forcing terms $\hat{\boldsymbol{F}}_{i j}^{2}$, and figures on the right show the real part of the associated response $\hat{w}_{i j}^{2}$. (a)-(b) Flow field of amplitude $A^{2}(m=2, \omega=0),(c)-(d)$ flow field of amplitude $B^{+} A^{*}\left(m=0, \omega=\omega_{0}\right)$ and $(e)-(f)$ flow field of amplitude $B^{+} B^{-*}(m=2$, $\omega=0)$. Note that the vertical scale is twice the horizontal scale.

zero frequency, shown in figure 6(a), whose associated response $\hat{\boldsymbol{q}}_{A A}^{2}$ is shown in figure $6(b)$. The advection of the spiralling mode of amplitude $B^{+}$by the stationary mode of amplitude $A^{*}$ (and vice versa) results in an axisymmetric forcing term beating at the frequency $\omega_{0}$. As seen in figures $6(c)$ and $6(d)$, the forcing term $\hat{\boldsymbol{F}}_{\boldsymbol{B}^{+} \boldsymbol{A}^{*}}$ and its associated response $\hat{\boldsymbol{q}}_{\boldsymbol{B}^{+} \boldsymbol{A}^{*}}^{2}$ are nearly periodic in space with a local wavelength close to that of the eigenmode $\hat{\boldsymbol{q}}_{\boldsymbol{B}^{+}}^{1}$. Finally, the advection of the spiralling mode of amplitude $B^{+}$by the corotating spiralling mode of amplitude $B^{-}$(and vice versa) generates a second-order forcing term $\hat{\boldsymbol{F}}_{\boldsymbol{B}^{+} \boldsymbol{B}^{-}}$of azimuthal wavenumber $m=2$ and zero frequency presented along with its associated response in figures $6(e)$ and $6(f)$.

\subsection{Order 3}

The problem at order $\epsilon^{3}$ is similar to that obtained at order $\epsilon^{2}$, as the third order solution $\boldsymbol{q}^{\mathbf{3}}$ obeys the forced linear Navier-Stokes equations

$$
\mathscr{B} \partial_{t} \boldsymbol{q}^{\mathbf{3}}+\tilde{\mathscr{A}}^{c} \boldsymbol{q}^{\mathbf{3}}=\boldsymbol{F}^{\mathbf{3}} \text {. }
$$

The forcing term $\boldsymbol{F}^{3}$ depends only on lower order solutions and reads

$$
\boldsymbol{F}^{\mathbf{3}}=-\left(\partial_{t_{1}} \boldsymbol{q}^{\mathbf{1}}-\mathscr{S} \boldsymbol{q}^{\mathbf{1}}+\delta \nabla^{2} \boldsymbol{u}^{\mathbf{1}}+\mathscr{C}\left(\boldsymbol{u}^{1}, \boldsymbol{u}^{\mathbf{2}}\right), 0\right)^{T},
$$

where $\mathscr{S}$ is the shift operator acting in the real space obtained from the operator $\mathscr{S}_{m}$ introduced in (4.4) by inverse Fourier transform in time and in the azimuthal 
direction. The first term $-\partial_{t_{1}} \boldsymbol{q}^{1}$ in (4.13) corresponds to the slow time evolution of the unknown amplitudes $A, B^{+}, B^{-}$, and will be referred to as the slow variation term. The second term $\mathscr{S} \boldsymbol{q}^{1}$ comes from the fact that, at the critical Reynolds number $R e_{c}$, the growth rates of the eigenmodes are zero at leading order, but depart from criticality at order $\epsilon^{2}$. To ease the discussion, this term will therefore be referred to as the off-criticality term. The third term $-\delta \nabla^{2} \boldsymbol{u}^{1}$ arises from the Reynolds number variation acting here on the order $\epsilon^{1}$ solution. The last term $-\mathscr{C}\left(\boldsymbol{u}^{1}, \boldsymbol{u}^{2}\right)$ is due to the advection of the first-order solution $\boldsymbol{q}^{1}$ by the second-order solution $\boldsymbol{q}^{2}$ and vice versa.

The first three forcing terms are linear and therefore resonate. The term $-\mathscr{C}\left(\boldsymbol{u}^{\mathbf{1}}, \boldsymbol{u}^{\mathbf{2}}\right)$ splits into two contributions: the linear term $-\mathscr{C}\left(\boldsymbol{u}^{1}, \boldsymbol{u}_{\delta}^{2}\right)$ takes into account the action of the Reynolds number variation through modifications of the axisymmetric base flow, and the nonlinear terms of various space and time periodicity generated through the combinations of the six contributions of the first-order solution together with the 21 other contributions of the second-order solution. Each of these terms is denoted $\hat{\boldsymbol{F}}_{i j}^{3} \mathrm{e}^{(\mathrm{i} m \theta+\mathrm{i} \omega t)}$, the subscripts $i$ (respectively $j$ ) standing for the amplitude of the firstorder (respectively second order) solution. Among these nonlinear forcing terms, many are resonant. This is for instance the case of the terms corresponding to the advection of the stationary mode $\hat{\boldsymbol{q}}_{\boldsymbol{A}}^{1}(m=1, \omega=0)$, by second-order contributions satisfying $(m=0, \omega=0)$ (see table 3 ). To avoid secular terms, or in other words, to be able to solve the expansion procedure at the third order, compatibility conditions have to be enforced using the Fredholm alternative (Friedrichs 1973). Specifically, the resonant forcing terms must be orthogonal to the kernel of the adjoint linearized Navier-Stokes operator. The compatibility conditions impose $A, B^{+}, B^{-}$to obey the relation

$$
\begin{aligned}
\frac{\mathrm{d} A}{\mathrm{~d} t_{1}} & =\frac{1}{\epsilon^{2}} \dot{A}=\tilde{\lambda}_{A} A-\tilde{\mu}_{A} A|A|^{2}-\tilde{v}_{A} A\left|B^{+}\right|^{2}-\tilde{v}_{A}^{*} A\left|B^{-}\right|^{2}-\tilde{\chi}_{A} B^{+} B^{-*} A^{*}, \\
\frac{\mathrm{d} B^{+}}{\mathrm{d} t_{1}} & =\frac{1}{\epsilon^{2}} \dot{B}^{+}=\tilde{\lambda}_{B} B^{+}-\tilde{\mu}_{B} B^{+}\left|B^{+}\right|^{2}-\tilde{v}_{B} B^{+}\left|B^{-}\right|^{2}-\tilde{\eta}_{B} B^{+}|A|^{2}-\tilde{\chi}_{B} B^{-} A^{2}, \\
\frac{\mathrm{d} B^{-}}{\mathrm{d} t_{1}} & =\frac{1}{\epsilon^{2}} \dot{B}^{-}=\tilde{\lambda}_{B} B^{-}-\tilde{\mu}_{B} B^{-}\left|B^{-}\right|^{2}-\tilde{v}_{B} B^{-}\left|B^{+}\right|^{2}-\tilde{\eta}_{B} B^{-}|A|^{2}-\tilde{\chi}_{B} B^{+} A^{* 2},
\end{aligned}
$$

which turns out to be identical to the normal form (1.2) if all coefficients in (1.2) are rescaled into their second-order counterparts defined as

$$
\left(\lambda_{A}, \mu_{A}, \ldots, \chi_{B}\right)=\epsilon^{2}\left(\tilde{\lambda}_{A}, \tilde{\mu}_{A}, \ldots, \tilde{\chi}_{B}\right) .
$$

The values of all complex coefficients of system (4.14) are computed as scalar products between the adjoint global modes $\hat{\boldsymbol{q}}^{1 \dagger}$ and the resonant forcing terms $\hat{\boldsymbol{F}}^{3}$. For instance the $\tilde{\chi}_{A}$ coefficient arises from a forcing term of amplitude $B^{+} B^{-*} A^{*}$, generated by three different $\boldsymbol{q}^{\mathbf{1}}-\boldsymbol{q}^{\mathbf{2}}$ interactions:

$$
\hat{\boldsymbol{F}}_{\boldsymbol{B}^{+} \boldsymbol{B}^{-*} \boldsymbol{A}^{*}}^{\mathbf{3}}=-\mathscr{C}_{1,0}\left(\hat{\boldsymbol{u}}_{\boldsymbol{B}^{+}}^{1}, \hat{\boldsymbol{u}}_{\boldsymbol{B}^{-} \boldsymbol{A}}^{2^{*}}\right)-\mathscr{C}_{1,0}\left(\hat{\boldsymbol{u}}_{\boldsymbol{B}^{-}}^{\mathbf{1}^{*}}, \hat{\boldsymbol{u}}_{\boldsymbol{B}^{+} \boldsymbol{A}^{*}}^{2}\right)-\mathscr{C}_{-1,2}\left(\hat{\boldsymbol{u}}_{\boldsymbol{A}}^{\mathbf{1}^{*}}, \hat{\boldsymbol{u}}_{\boldsymbol{B}^{+} \boldsymbol{B}^{-*}}^{\mathbf{2}}\right) .
$$

It can be easily checked that, for instance, the interaction between the spiralling mode $\hat{\mathbf{q}}_{\mathbf{B}^{+}}^{1}\left(m=1, \omega=\omega_{0}\right)$, and the axisymmetric response $\hat{\boldsymbol{u}}_{\boldsymbol{B}^{-}} \boldsymbol{A}^{*}\left(m=0, \omega=-\omega_{0}\right)$ is indeed resonant with the stationary mode $\hat{\boldsymbol{u}}_{\boldsymbol{A}}^{\mathbf{1}}$. The axial velocity component of $\hat{\boldsymbol{F}}_{\boldsymbol{B}^{+} \boldsymbol{B}^{-\boldsymbol{A}^{*}}}^{\mathbf{*}}$ is presented in figure $7(a)$. This forcing is extended downstream but reaches a maximum in the recirculating bubble. Since $\left\langle\hat{\boldsymbol{q}}_{\boldsymbol{A}}^{1 \dagger}, \mathscr{B} \hat{\boldsymbol{q}} \hat{\boldsymbol{A}}^{1}\right\rangle=1$ with the present normalization, $\tilde{\chi}_{A}$ is 

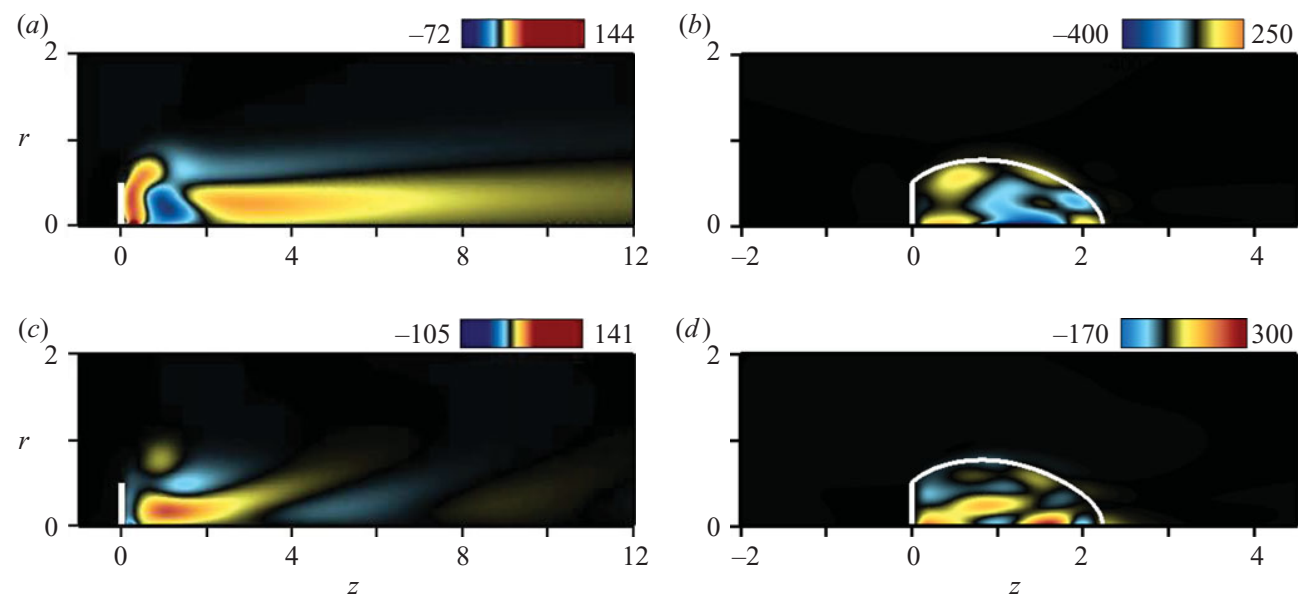

FIGURE 7. Representation of various resonant forcing fields appearing at order $\epsilon^{3}$ in the weakly nonlinear analysis. (a) Axial component of the forcing term of amplitude $B^{+} B^{-*} A^{*}$ responsible for coefficient $\tilde{\chi}_{A}$ in equation (4.14a). (b) Coupling density field $\tilde{\chi}_{A}(r, z)$, defined as the scalar product between this forcing term and the adjoint global mode $\hat{\boldsymbol{q}}_{A}^{\mathbf{1}^{\dagger}}$. (c) Real part of the axial component of the forcing term of amplitude $B^{-} A^{2}$ responsible for coefficient $\tilde{\chi}_{B}$ in $(4.14 b)$ and $(4.14 c)$. (d) Real part of the coupling density field $\tilde{\chi}_{B}(r, z)$, defined as the scalar product between this forcing term and the adjoint global mode $\hat{\boldsymbol{q}}_{\boldsymbol{B}^{+}}^{\mathbf{1}^{\dagger}}$. Note that the vertical scale is twice the horizontal scale in figures $7(a)$ and $7(c)$.

given by

$$
\tilde{\chi}_{A}=-\left\langle\hat{\boldsymbol{q}}_{A}^{1 \dagger}, \hat{\boldsymbol{F}}_{\boldsymbol{B}^{+} \boldsymbol{B}^{-*} A^{*}}^{3}\right\rangle .
$$

It can be seen from (4.17) that the value of the second-order coefficient $\tilde{\chi}_{A}$ is independent of the choice of $\epsilon$, giving that the nonlinear coefficients of (1.2) should scale as $\epsilon^{2}$. This is a well-known result, since the precise value of the nonlinear coefficients in a normal form depends on the normalization of the modes amplitudes. Consequently, all results will be hereinafter presented in terms of the second-order coefficients. Figure $7(b)$ shows the coupling density $\tilde{\chi}_{A}(r, z)=\hat{\boldsymbol{q}}_{A}^{\dagger \dagger}(r, z) \cdot \hat{\boldsymbol{F}}_{\boldsymbol{B}^{+} \boldsymbol{B}^{-*} \boldsymbol{A}^{*}}(r, z)$, such that the coupling coefficient $\tilde{\chi}_{A}$ reads $\tilde{\chi}_{A}=\int_{\Omega} \tilde{\chi}_{A}(r, z) r \mathrm{~d} r \mathrm{~d} z$. One observes that the coupling density $\tilde{\chi}_{A}(r, z)$ vanishes outside the recirculation bubble, since the adjoint global mode is localized within the recirculation bubble and upstream of the disk (figure $4 b$ ), and the nonlinear forcing $\hat{\boldsymbol{F}}_{\boldsymbol{B}^{+} \boldsymbol{B}^{\boldsymbol{B}^{*} \boldsymbol{A}^{*}}}$ is localized downstream (figure $7 a$ ). This indicates that the resonant forcing of mode $\hat{\boldsymbol{q}}_{A}^{1}$ owing to the $B^{+} B^{-*} A^{*}$ interaction is efficient only in the recirculation bubble. It is worthwhile emphasizing that this result can be generalized to all nonlinear coefficients of the normal form (4.14), that are computed as the scalar product of a forcing term localized downstream of the disk, with an adjoint global mode localized upstream of the disk and in the recirculation bubble. They are therefore only determined by a coupling that occurs within the recirculation bubble. The recirculation region may therefore be viewed as the effective wavemaker since all nonlinear interactions between the instability modes take place in this region. Even though nonlinear forcing terms and nonlinear responses are all spatially extended downstream, the region outside the recirculation bubble may be viewed as passive since values of the flow field there do not influence the dynamics of the leading modes. 


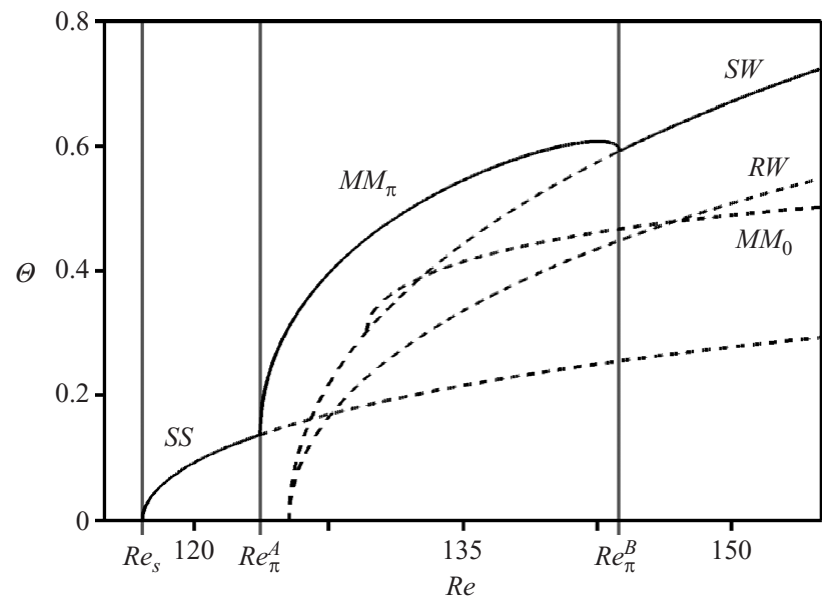

FIGURE 8. Theoretical bifurcation diagram associated to the normal form (4.14). Solid (respectively dashed) lines denote stable (respectively unstable) branches.

Similarly, $\tilde{\chi}_{B}$ results from a forcing term of amplitude $B^{-} A A$, whose real part of the axial velocity component is shown in figure $7(c)$. It arises from different contributions:

$$
\begin{aligned}
\hat{\boldsymbol{F}}_{\boldsymbol{B}^{-} \boldsymbol{A A}}^{\mathbf{3} A} & =-\mathscr{C}_{1,0}\left(\hat{\boldsymbol{u}}_{\boldsymbol{A}}^{\mathbf{1}}, \hat{\boldsymbol{u}}_{\boldsymbol{B}^{-} \boldsymbol{A}}^{\mathbf{A}}\right)-\mathscr{C}_{-1,2}\left(\hat{\boldsymbol{u}}_{\boldsymbol{B}^{-}}^{\mathbf{1}}, \hat{\boldsymbol{u}}_{\boldsymbol{A} \boldsymbol{A}}^{\mathbf{2}}\right), \\
\tilde{\chi}_{B} & =-\left\langle\hat{\boldsymbol{q}}_{B^{+}}^{\mathbf{1}}, \hat{\boldsymbol{F}}_{B^{-} \boldsymbol{A A}}^{\mathbf{3}}\right\rangle .
\end{aligned}
$$

This means that the spiralling mode $\hat{\boldsymbol{q}}_{\boldsymbol{B}^{-}}^{\mathbf{1}}$ can force the production of the counterrotating spiralling mode $\hat{\boldsymbol{q}}_{\boldsymbol{B}^{+}}^{\mathbf{1}}$ by its nonlinear interaction with the $m=2$ stationary solution $\hat{\boldsymbol{q}}_{\boldsymbol{A} \boldsymbol{A}}^{\mathbf{2}}$ shown in figure $6(b)$, or by interacting first with the stationary mode $\hat{\boldsymbol{q}}_{\boldsymbol{A}}^{\mathbf{1}}$ to produce the second-order solution $\hat{\boldsymbol{q}}_{\boldsymbol{B}^{-} \boldsymbol{A}}^{\mathbf{A}}\left(m=0, \omega=\omega_{0}\right)$, which eventually interacts with $\hat{\boldsymbol{q}}_{A}^{\mathbf{1}}$ again. The real part of the associated coupling density field $\tilde{\chi}_{B}(r, z)=$ $\hat{\boldsymbol{q}}_{\boldsymbol{B}^{+}}^{\mathbf{1}}(r, z) \cdot \hat{\boldsymbol{F}}_{\boldsymbol{B}^{-} \boldsymbol{A A A}_{\boldsymbol{A}}}(r, z)$, shown in figure $7(d)$, is also localized in the recirculation region, meaning that the spiralling mode $\hat{\boldsymbol{q}}_{\boldsymbol{B}^{+}}^{\mathbf{1}}$ is receptive to the forcing owing to the $B^{-} A A$ interaction only close to the disk and in the recirculating bubble.

We obtain

$$
\begin{aligned}
& \tilde{\lambda}_{A}=2.01+71.4 \delta \\
& \tilde{\mu}_{A}=3.11 \\
& \tilde{\nu}_{A}=6.88-1.11 \mathrm{i} \\
& \tilde{\chi}_{A}=4.57
\end{aligned}
$$$$
\begin{aligned}
& \tilde{\lambda}_{B}=-1.85+76.4 \mathrm{i}+(66.7+9.35 \mathrm{i}) \delta \\
& \tilde{\mu}_{B}=2.42+0.0321 \mathrm{i} \\
& \tilde{\nu}_{B}=3.13-0.816 \mathrm{i} \\
& \tilde{\eta}_{B}=0.955-3.47 \mathrm{i} \\
& \tilde{\chi}_{B}=1.62-1.36 \mathrm{i} .
\end{aligned}
$$

\subsection{Bifurcation diagram}

We set now $A=|A| \mathrm{e}^{\mathrm{i} \phi_{\mathrm{A}}}, B^{+}=\left|B^{+}\right| \mathrm{e}^{\mathrm{i} \phi_{\mathrm{B}}^{+}}$and $B^{-}=\left|B^{-}\right| \mathrm{e}^{\mathrm{i} \phi_{\mathrm{B}}^{-}}$. An exhaustive description of the solutions of system (4.14), up to ternary bifurcations, can be found in Golubitsky et al. (1988). In this section, we comment only the solutions relevant to our problem. The bifurcation diagram is shown in figure 8 , where the quantity $\Theta=|A|+\left|B^{+}\right|+\left|B^{-}\right|$ is plotted as a function of the Reynolds number. Note that $\Theta$ has no particular physical meaning but yields a convenient visualization of the bifurcation sequence. The solid thick lines (respectively thin dashed lines) correspond to stable (respectively unstable) solutions that are of three different kinds, as will now be explained. 


\subsubsection{Pure modes}

In addition to the trivial steady axisymmetric solution $\left(A, B^{+}, B^{-}\right)=(0,0,0)$, equations (4.14) have three types of solutions involving a single mode. The pure steady state $S S(A, 0,0)$ is ruled by the real Landau equation for $A$ :

$$
\dot{A}=\epsilon^{2}\left(\tilde{\lambda}_{A}-\tilde{\mu}_{A} A|A|^{2}\right),
$$

so that $A^{2}=|A|^{2}=\tilde{\lambda}_{A} / \tilde{\mu}_{A}$ and $\dot{\phi}_{\mathrm{A}}=0$, and we can impose $\phi_{\mathrm{A}}=0$ by choosing the phase of the initial disturbance to be zero. The pure Hopf states $R W\left(0, B^{+}, 0\right)$ and $\left(0,0, B^{-}\right)$are associated with spiralling modes, whose amplitude is governed by the complex Landau equation for $B^{ \pm}$:

$$
\dot{B}^{ \pm}=\epsilon^{2}\left(\tilde{\lambda}_{B} B^{ \pm}-\tilde{\mu}_{B} B^{ \pm}\left|B^{ \pm}\right|^{2}\right)
$$

so that

$$
\begin{aligned}
\left|B^{ \pm}\right|^{2} & =\frac{\tilde{\lambda}_{B r}}{\tilde{\mu}_{B r}}, \\
\phi_{\mathrm{B}}^{ \pm} & =\epsilon^{2}\left(\tilde{\lambda}_{B i}-\tilde{\mu}_{B i}\left|B^{ \pm}\right|^{2}\right) t+\phi_{\mathrm{B}}^{ \pm^{0}} .
\end{aligned}
$$

The standing wave $S W\left(0, B^{+}, B^{-}\right)$corresponds to the superimposition of two counter-rotating spiralling modes of same amplitude $\left|B^{ \pm}\right|^{2}$ governed by the amplitude equation

$$
\dot{B}^{ \pm}=\epsilon^{2}\left(\tilde{\lambda}_{B} B^{ \pm}-\left(\tilde{\mu}_{B}+\tilde{v}_{B}\right) B^{ \pm}\left|B^{ \pm}\right|^{2}\right)
$$

of solutions

$$
\begin{aligned}
\left|B^{ \pm}\right|^{2} & =\frac{\tilde{\lambda}_{B r}}{\tilde{\mu}_{B r}+\tilde{v}_{B r}}, \\
\phi_{\mathrm{B}}^{ \pm}(t) & =\epsilon^{2}\left(\tilde{\lambda}_{B i}-\left(\tilde{\mu}_{B i}+\tilde{v}_{B i}\right)\left|B^{ \pm}\right|^{2}\right) t+{\phi_{\mathrm{B}}^{ \pm}}^{0} .
\end{aligned}
$$

\subsubsection{Mixed modes}

Mixed modes $M M\left(A, B^{+}, B^{-}\right)$correspond to a superposition of the three global modes, i.e. one stationary mode and two counter-rotating spiral modes of same amplitude $\left|B^{ \pm}\right|$. Introducing the phase $\phi=\phi_{\mathrm{B}}^{+}-\phi_{\mathrm{B}}^{-}-2 \phi_{\mathrm{A}}$ allows to reduce system (4.14) to a three-dimensional polar system for $|A|,\left|B^{ \pm}\right|$and $\phi$ that reads:

$$
\begin{aligned}
|\dot{A}| & =\epsilon^{2}\left(\tilde{\lambda}_{A}|A|-\tilde{\mu}_{A}|A|^{3}-\left(2 \tilde{v}_{A r}+\tilde{\chi}_{A} \cos \phi\right)|A|\left|B^{ \pm}\right|^{2}\right), \\
\left|\dot{B^{ \pm}}\right| & =\epsilon^{2}\left(\tilde{\lambda}_{B r}\left|B^{ \pm}\right|-\left(\tilde{\mu}_{B r}+\tilde{v}_{B r}\right)\left|B^{ \pm}\right|^{3}-\left(\tilde{\eta}_{B r}+\tilde{\chi}_{B r} \cos \phi \pm \tilde{\chi}_{B i} \sin \phi\right)\left|B^{ \pm}\right||A|^{2}\right), \\
\dot{\phi} & =2 \epsilon^{2}\left(\tilde{\chi}_{B r}|A|^{2}+\tilde{\chi}_{A}\left|B^{ \pm}\right|^{2}\right) \sin \phi .
\end{aligned}
$$

The phase $\phi$ is thus solution of $\sin \phi=0$, so that it comes from $(4.24 c)$ that $\phi$ is invariant. The solutions of system (4.24) are such that

$$
\begin{aligned}
\cos \phi & = \pm 1, \\
|A|^{2} & =\frac{\tilde{\lambda}_{A}\left(\tilde{\mu}_{B r}+\tilde{\nu}_{B r}\right)-\left(2 \tilde{v}_{A r}+\cos \phi \tilde{\chi}_{A}\right) \tilde{\lambda}_{B r}}{\tilde{\mu}_{A}\left(\tilde{\mu}_{B r}+\tilde{v}_{B r}\right)-\left(2 \tilde{\nu}_{A r}+\cos \phi \tilde{\chi}_{A}\right)\left(\tilde{\eta}_{B r}+\cos \phi \tilde{\chi}_{B r}\right)}, \\
\left|B^{ \pm}\right|^{2} & =\frac{-\tilde{\lambda}_{A}\left(\tilde{\eta}_{B r}+\cos \phi \tilde{\chi}_{B r}\right)+\tilde{\mu}_{A} \tilde{\lambda}_{B r}}{\tilde{\mu}_{A}\left(\tilde{\mu}_{B r}+\tilde{v}_{B r}\right)-\left(2 \tilde{v}_{A r}+\cos \phi \tilde{\chi}_{A}\right)\left(\tilde{\eta}_{B r}+\cos \phi \tilde{\chi}_{B r} r\right.}, \\
\phi_{B}^{ \pm}(t) & =\epsilon^{2}\left(\tilde{\lambda}_{B i}-\left(\tilde{\mu}_{B i}+\tilde{v}_{B i}\right)\left|B^{ \pm}\right|^{2}-\left(\tilde{\eta}_{B i}+\tilde{\chi}_{B i} \cos \phi\right)|A|^{2}\right) t \pm\left(\phi+2 \phi_{A}\right) / 2,
\end{aligned}
$$



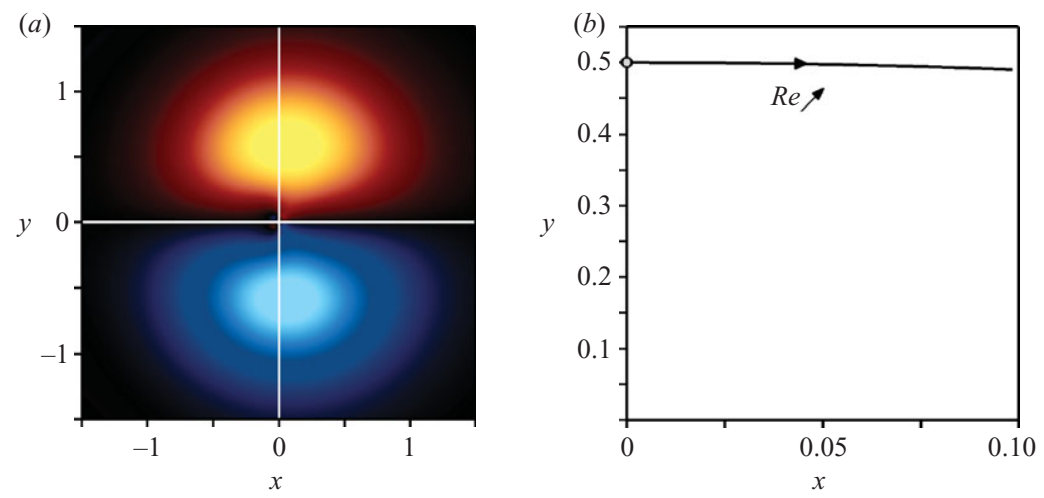

FiguRE 9. Streamwise vorticity of the SS mode. (a) Cross-sectional contours of streamwise vorticity, computed at the bifurcation threshold $R e_{c}^{A}$, at the axial position $z=20$. (b) For the same axial position, trajectory of the vortex core in the $(x, y)$ plane as the Reynolds number is increased in the range $117.1<R e<123$, for the solution computed up to the second order. This lateral shift of the longitudinal vortex dipole is due to the self-induction of the perturbation, accounted for by the second harmonic term $\hat{\boldsymbol{q}}_{\boldsymbol{A} A}^{2}$ in the asymptotic expansion (4.10).

so that the mixed modes come in two different states, referred to as $M M_{0}(\cos \phi=1)$ and $M M_{\pi}(\cos \phi=-1)$ respectively. Note that since coefficient $\tilde{\chi}_{A}$ is real, $(4.14 a)$ leads to $\dot{\phi}_{\mathrm{A}}=0$, so that we can again impose $\phi_{\mathrm{A}}=0$ without particularizing the solution of the system.

\subsection{Bifurcating modes}

Unless otherwise specified, all flow fields presented in this section refer to the secondorder nonlinear solution $\boldsymbol{q}=\boldsymbol{q}^{\mathbf{0}}+\epsilon \boldsymbol{q}^{\mathbf{1}}+\epsilon^{2} \boldsymbol{q}^{\mathbf{2}}$, the term of order $\epsilon^{2}$ being essential to discuss some realistic features. For the present coefficient values, the domains of existence and the stability of the different modes are shown in figure 8:

(a) For low Reynolds numbers $R e<117.1$, the trivial steady axisymmetric solution $\Theta=0$ is stable. It exhibits an infinite number of symmetry planes.

(b) The first bifurcation occurs at $R_{s}=117.1$, where the trivial state bifurcates to the pure steady state $S S$. The threshold of this bifurcation, for which $\tilde{\lambda}_{A}=0$, departs slightly from the critical Reynolds number issuing from the direct stability analysis $R e_{c}^{A}$, as it is obtained by considering the linear approximation of the growth rate. As discussed previously, the stationary mode that bifurcates corresponds to a shift of the wake in one direction, associated with the appearance of a pair of longitudinal vortices. If one only looks at the first-order solution, the longitudinal vortex dipole is centred, but the asymptotic second-order solution allows to describe the off-axis displacement of the dipole under its self-induction (figures $9 a$ and $9 b$ ). Figure $9(a)$ shows cross-sectional contour plots of the streamwise vorticity at a Reynolds number $R e=118.5$ above threshold, the section being taken 20 diameters downstream of the disk. We observe a counter-rotating vortex dipole which is reminiscent of that observed experimentally in axisymmetric wakes in this regime (Thompson, Leweke \& Provansal 2001), and usually designated as 'vortex threads'. Note that the cores of the vortex dipole, defined as the points of extremal streamwise vorticity of $\boldsymbol{q}$, are located off the centreplane $x=0$ at threshold, as each vortex induces a velocity at the centreline of the other, hence causing them to be convected away from the centreplane. This nonlinear effect is shown in figure $9(b)$, where the position of the vortex core in the $(x, y)$ plane is plotted as a function of the Reynolds number. The vertical 

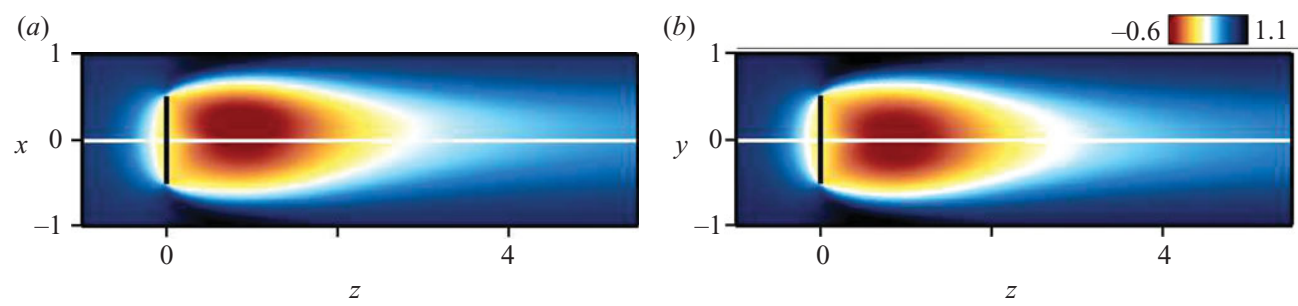

(c)

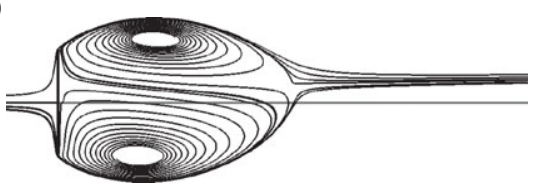

(d)

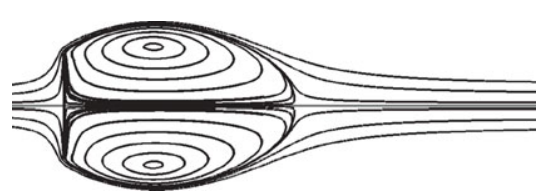

Figure 10. $(a)-(b)$ Streamwise velocity $\hat{w}$ and $(c)-(d)$ streamlines of the $S S$ solution, expanded up to the second order (i.e. $\left.\boldsymbol{q}=\boldsymbol{q}^{\mathbf{0}}+\epsilon \boldsymbol{q}^{1}+\epsilon^{2} \boldsymbol{q}^{2}\right)$ at $\boldsymbol{R e}=123$. (a) $-(c)(x, z)$ plane and $(b)-(d)$ $(y, z)$ plane.

(a)

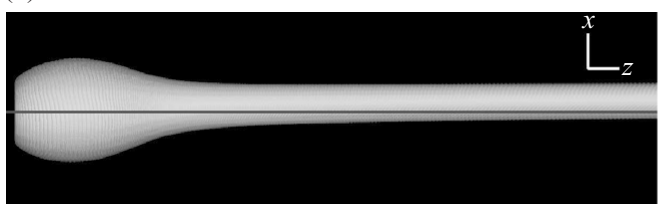

(b)

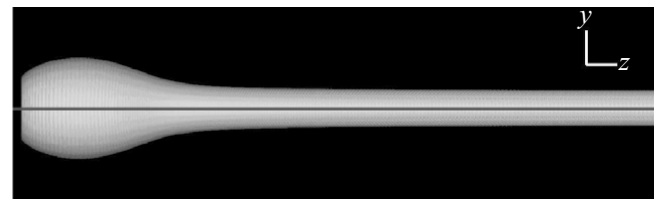

FIGURE 11. Numerically computed dye lines corresponding to the second-order SS solution shown in figure 10. The dark grey line represents the revolution axis and the wake has moved off-axis only in the $(x, z)$ side view.

position of the core barely changes, whereas the maximum horizontal deviation is zero at threshold and increases when the Reynolds number is increased, i.e. when the amplitude of the perturbation is increased. It finally reaches approximately 0.1 disk diameter for $R e=123$. In the present asymptotic expansion procedure, this effect is taken into account through the second-order term $\mathscr{C}_{1,1}\left(\hat{\boldsymbol{q}}_{A}^{\mathbf{1}}, \hat{\boldsymbol{q}}_{A}^{\mathbf{1}}\right)$ corresponding to the generation of the $m=2$ harmonic $\hat{\boldsymbol{q}}_{\boldsymbol{A} A}^{2}$. Indeed, when the nonlinear contribution of this harmonic is removed, the vortex dipole remains on the $y$-axis (grey circular symbol in figure $9 b$ ).

When considering the flow field, these vortex threads break the reflectional symmetry with respect to the $(y, z)$ plane but preserve that with respect to the $(x, z)$ plane, as evidenced by the axial velocity fields of the saturated flow field shown in figure 10: one thus observes that figures $10(a)$ and $10(c)$, showing the $(x, z)$ plane, are not symmetric with respect to the $(y, z)$ plane, whereas figures $10(b)$ and $10(d)$, showing the $(y, z)$ plane, are symmetric with respect to the $(x, z)$ plane. The breaking of the rotational symmetry is also evidenced in figure 11, which simulates an experimental dye visualization and shows numerically computed dye lines emitted from the disk surface and transported by the second-order analytic solution. Such visualization has been obtained by following the displacement of 360 particles emitted from $t=0$ to $t=30$ at the position $r=0.4$ and $z=0.01$, with a uniform distribution in azimuth. Note that the symmetry plane can be arbitrarily rotated as the phase $\phi_{\mathrm{A}}$ has been here arbitrarily selected. 
(a)

$x$

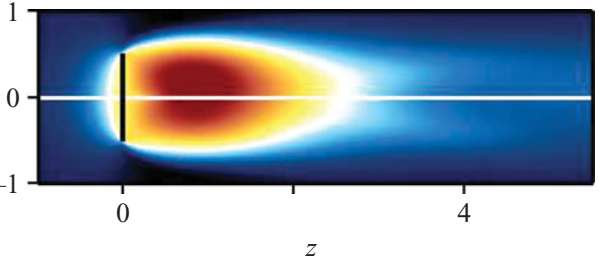

(b)

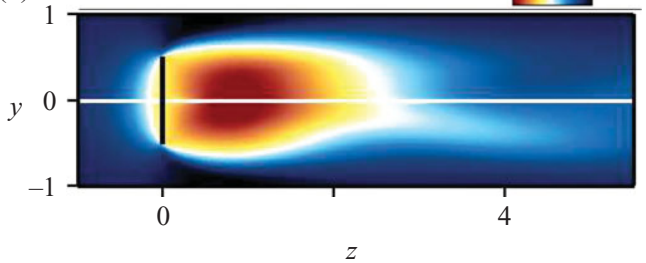

FIGURE 12. Flow after the second bifurcation: axial velocity $w$ of the $M M_{\pi}$ solution, expanded up to the second order $-R e=136$. $(a)(x, z)$ plane and $(b)(y, z)$ plane.

(a)

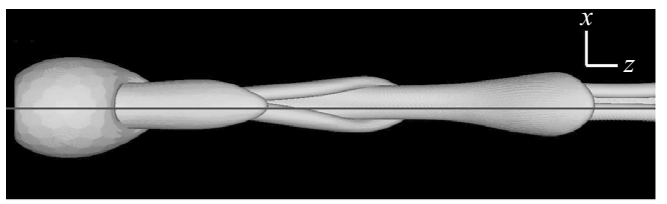

(c)

(b)
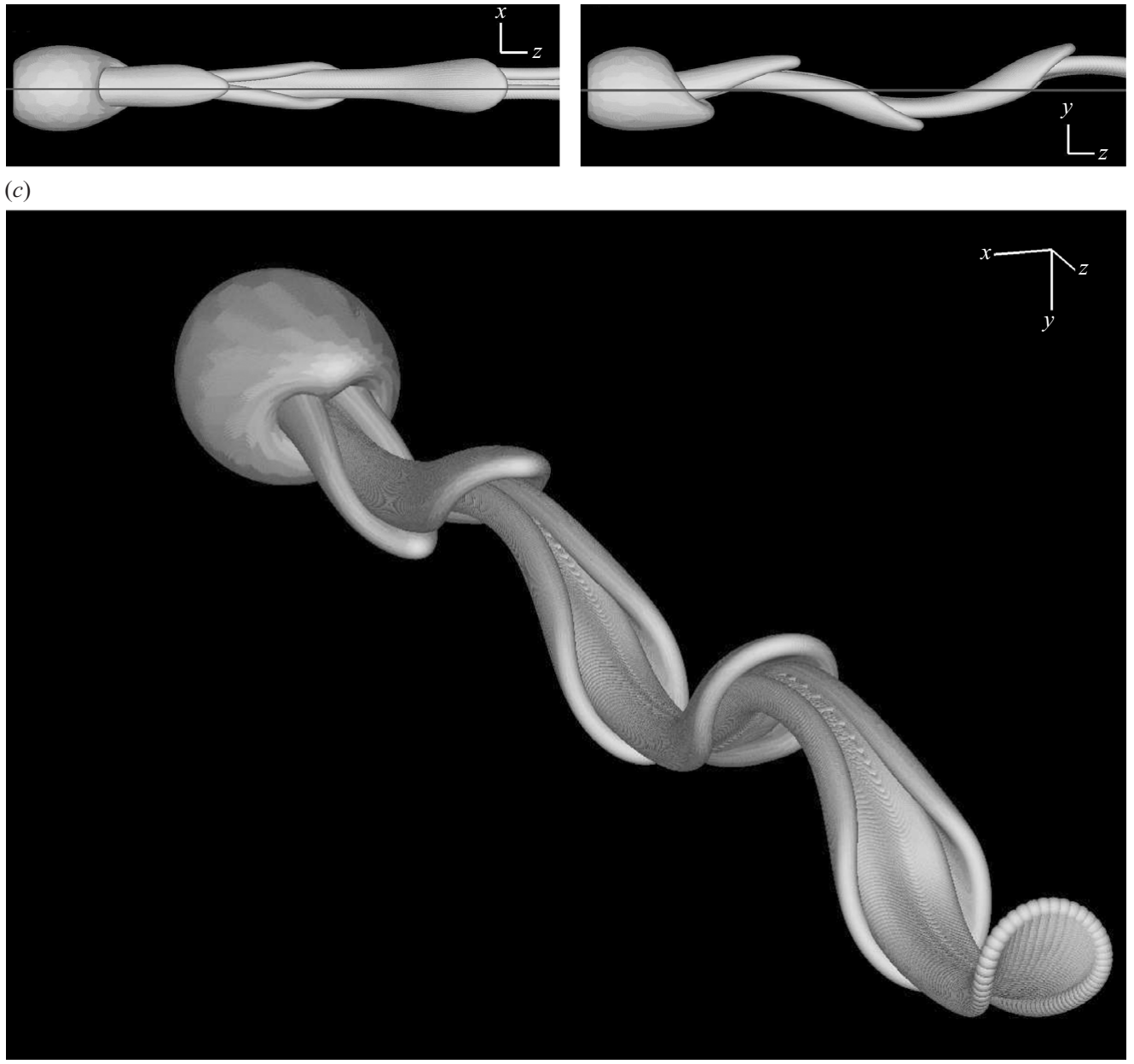

FIGURE 13. Numerically computed dye lines based on the asymptotic expansion up to the second order, corresponding to the $M M_{\pi}$ solution shown in figure 12 . $(a)(x, z)$ plane: the $x \longrightarrow-x$ symmetry is broken by a drift of the wake towards positive values of $x$ downstream of the disk, $(b)(y, z)$ plane and $(c)$ three-dimensional representation. 


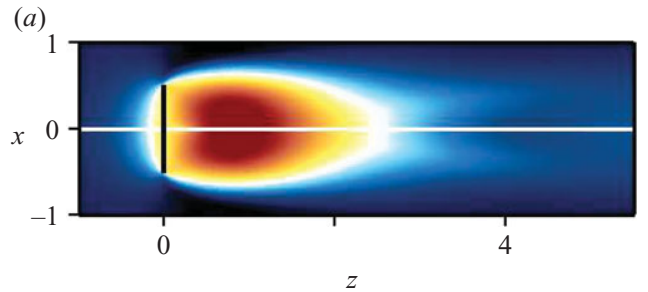

(b)

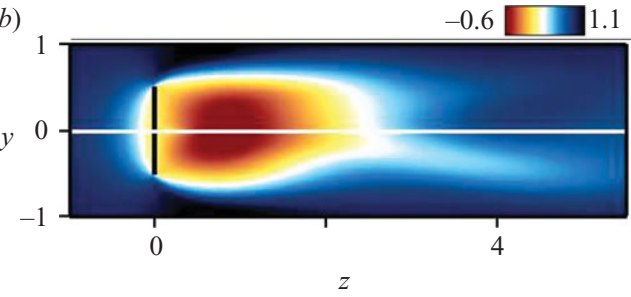

FIGURE 14. Flow after the third bifurcation: axial velocity $w$ of the $S W$ solution, expanded up to the second order $-R e=144$. $(a)(x, z)$ plane and $(b)(y, z)$ plane.

(a)

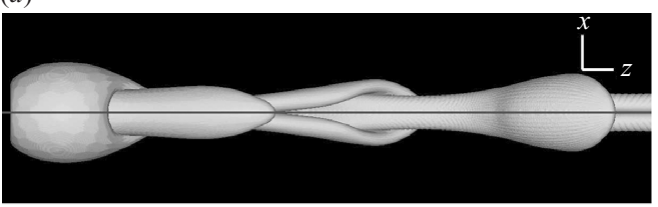

(b)

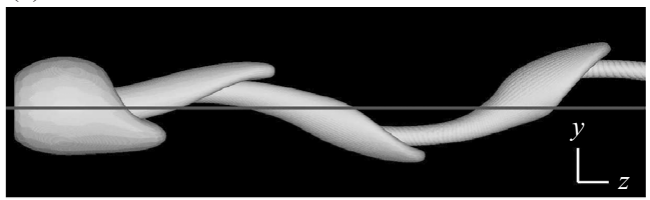

(c)

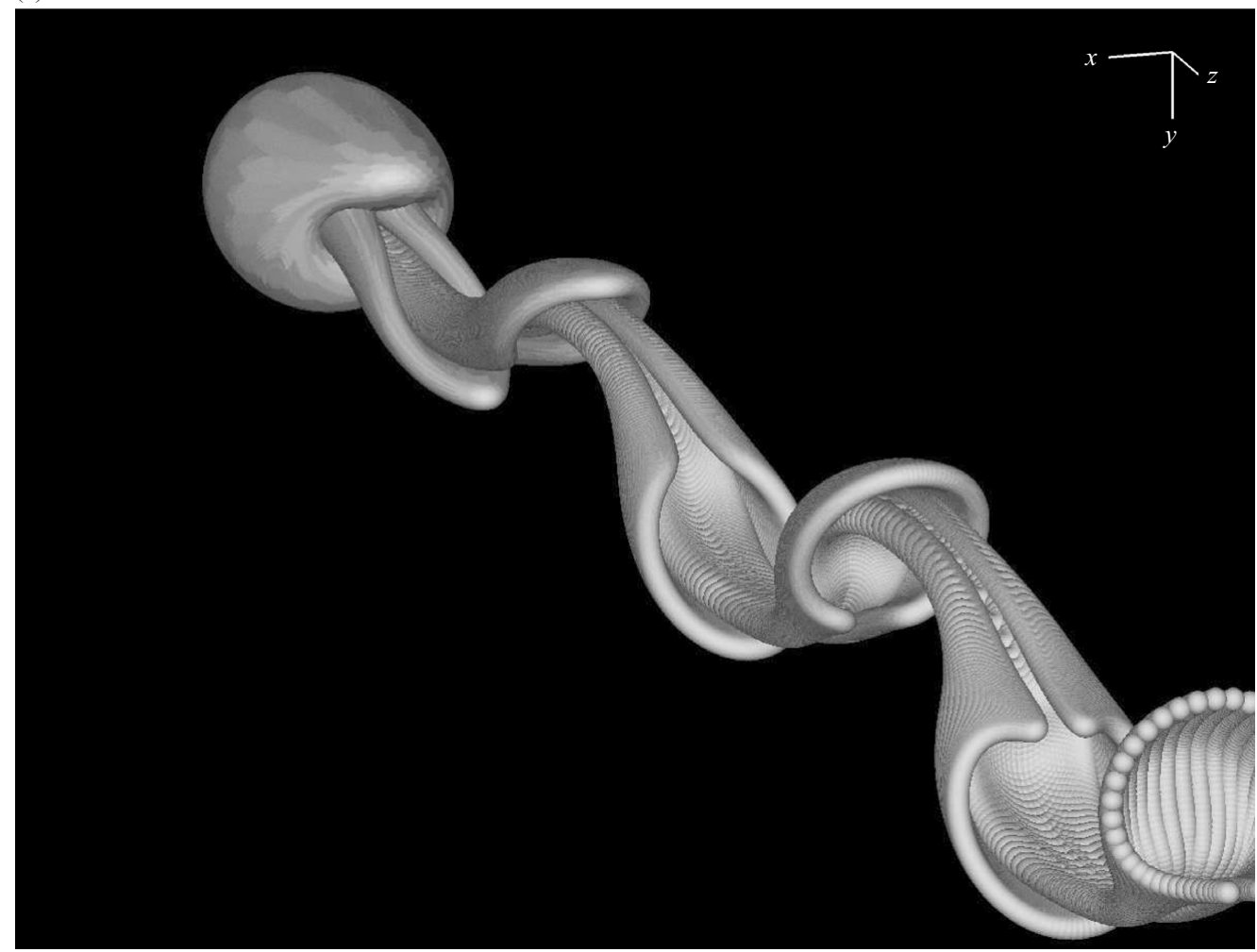

FIGURE 15. Numerical dye lines corresponding to the $S W$ solution shown in figure 14. $(a)(x, z)$ plane: the $x \longrightarrow-x$ symmetry is recovered, $(b)(y, z)$ plane and $(c)$ three-dimensional representation.

(c) A second bifurcation occurs at $R e_{\pi}^{A}=123.7$, where the $S S$ state bifurcates to the $M M_{\pi}$ branch where $\cos \phi=-1(\phi=\pi)$. At threshold, the $S S$ branch loses its stability to disturbances of small amplitudes $\left|B^{+}\right|=\left|B^{-}\right|=\left|B^{ \pm}\right|$, their second-order growth rate 
being given by

$$
\tilde{\sigma}_{\pi}^{A}=\tilde{\lambda}_{B r}-\left(\tilde{\eta}_{B r}-\tilde{\chi}_{B r}\right) \frac{\tilde{\lambda}_{A}}{\tilde{\mu}_{A}}
$$

In the present case, the mode of invariant phase $\phi=\pi$ destabilizes first the $S S$ branch since $\tilde{\chi}_{B r}$ is positive (see Golubitsky et al. 1988, for more details) and the Hopf bifurcation from the $S S$ branch occurs in favour of the $M M_{\pi}$ mode. The resulting pattern breaks the reflectional symmetry of the $S S$ mode with a periodic flapping of the wake in the $(y, z)$ plane, associated with a shedding of vortices, as seen in figure 12 , showing the axial velocity component in the $(x, z)$ and $(y, z)$ planes for the Reynolds number $R e=136$. The dye lines computed using the periodic flow given by the second-order asymptotic expansion are shown in figure 13, and illustrate the loss of all symmetries of the flow. Moreover, figure 13 demonstrates the ability of such asymptotic expansion to represent complex flows with dye lines exhibiting realistic knitted hairpin structures. Were $\tilde{\chi}_{B r}$ negative, then the $S S$ mode would have bifurcated to the $M M_{0}$ branch. In the present case, this branch is not selected and is unstable. Still, the corresponding numerical dye lines are shown in figure 16 for comparison at the same Reynolds number $R e=136$, although one should keep in mind that the resulting flow is unstable and should not be observed.

(d) A third bifurcation occurs at $R e_{\pi}^{B}=143.7$, where the $M M_{\pi}$ branch bifurcates to the $S W$ branch, where $A=0$. The critical Reynolds number is found by considering the backward bifurcation from the $S W$ to the $M M_{\pi}$ states, i.e. by studying how the $S W$ branch loses its stability to disturbances of small amplitude $|A|$, whose second-order growth rate is given by

$$
\tilde{\sigma}_{\pi}^{B}=\tilde{\lambda}_{A}-\left(2 \tilde{\nu}_{A r}-\tilde{\chi}_{A}\right) \frac{\tilde{\lambda}_{B r}}{\tilde{\mu}_{B r}+\tilde{\nu}_{B r}} .
$$

As reported in Golubitsky et al. (1988), the $S W$ branch, rather than the $R W$ branch, is selected here, since $\tilde{v}_{B r}>\tilde{\mu}_{B r}$. The condition for this bifurcation to occur is $\tilde{\chi}_{A}>0$, i.e. the development of the standing wave must restabilize the stationary eigenmode $\hat{\boldsymbol{q}}_{A}^{1}$, which is the case here. Views of the axial velocity component at the Reynolds number $R e=144$ are shown in figure 14, and the associated numerical dye lines at this Reynolds number are shown in figure 15. Comparing to the analogous representation of the $M M_{\pi}$ branch shown in figures 12 and 13, the difference may appear subtle, but the symmetry with respect to the $(y, z)$ plane is recovered, as shown in figures $14(a)$ and 15(a), whereas the wake was shifted up in figures $12(a)$ and $13(a)$.

\subsection{Comparison with the DNS calculations}

DNS of the wake of a circular disk placed normal to a uniform flow have been performed by Fabre et al. (2008). In this study, it has been observed that, as the Reynolds number is increased, the flow undergoes a sequence of successive bifurcations, each state being characterized by specific time and space symmetry breaking or recovering. These authors report a first bifurcation at the Reynolds number $R e_{s} \simeq 115$, leading to a steady state with a reflectional symmetry, corresponding to the present $S S$ branch. Then, a Hopf bifurcation is found for $R e_{\pi}^{A} \simeq 121$, leading to a so-called reflectional symmetry breaking state, characterized by the periodic shedding of vortices twisted around the symmetry axis, with no symmetry plane. The bifurcation threshold and symmetry properties therefore agree with that of the present $M M_{\pi}$ branch. Finally, a third bifurcation is observed for $R e_{\pi}^{B} \simeq 140$ and allows the flow to recover a planar symmetry, the recovered symmetry plane being 
(a)

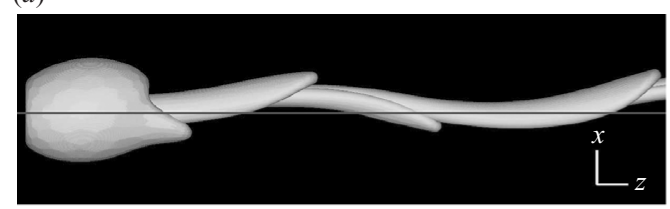

(b)

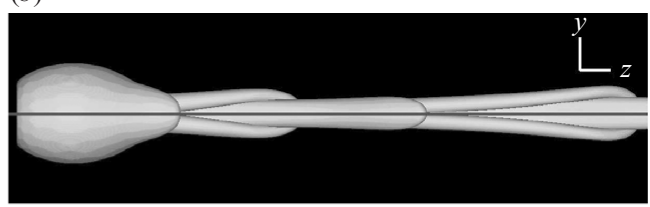

(c)

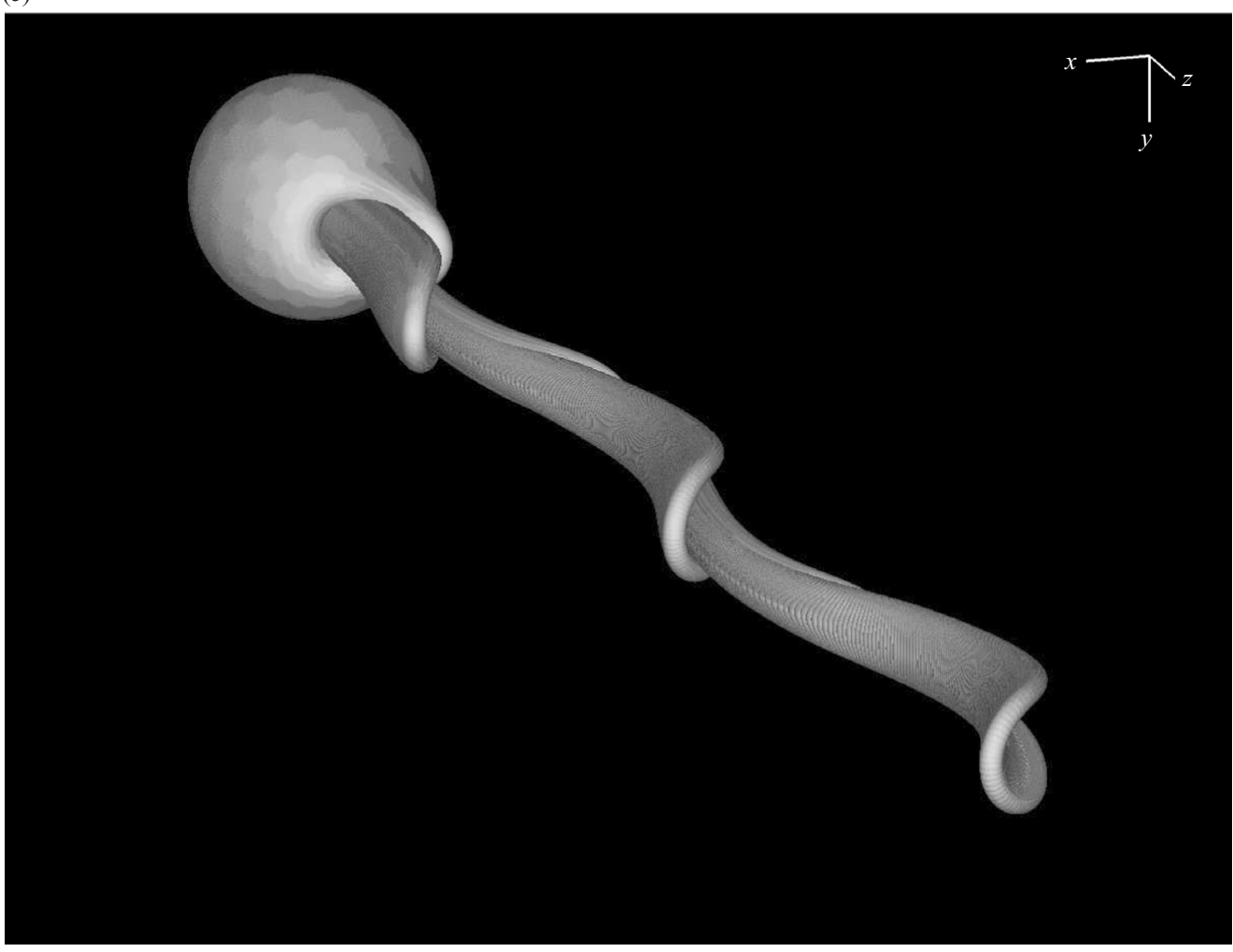

FIGURE 16. Numerical dye lines corresponding to the unstable $M M_{0}$ solution expanded up to the second order $-R e=136$. $(a)(x, z)$ plane, $(b)(y, z)$ plane and $(c)$ three-dimensional representation. It turns out that interestingly, this mode preserves the reflectional symmetry of the $S S$ solution. The result is reminiscent of the dye lines pattern observed experimentally in the wake past spheres, as in Gumowski et al. (2008), for instance. Note that the question of symmetry preserving/breaking in sphere flows has also been addressed in the recent study of Pier (2008).

found to be orthogonal to that initially selected in the steady state. This bifurcation threshold and symmetry properties agree with that of the present $S W$ branch.

The whole bifurcation sequence, including the third bifurcation, predicted by the present asymptotic expansion matches qualitatively and quantitatively for threshold values with that found in the DNS calculations. Note that as mentioned in $\S 1$, there exist other techniques to compute the coefficients of the normal form (4.14). For instance, one could investigate experimentally or numerically the transient dynamics at various Reynolds numbers. In the case of the cylinder wake, such procedure has allowed Provansal et al. (1987) to compute with accuracy the Landau coefficient associated with the Hopf bifurcation. Though, such methods can be expected to also yield results in good agreement with that presented here, since we show in appendix B that the sensitivity of the bifurcation diagram to small variations in the different coefficients is remarkably small. 
(a)

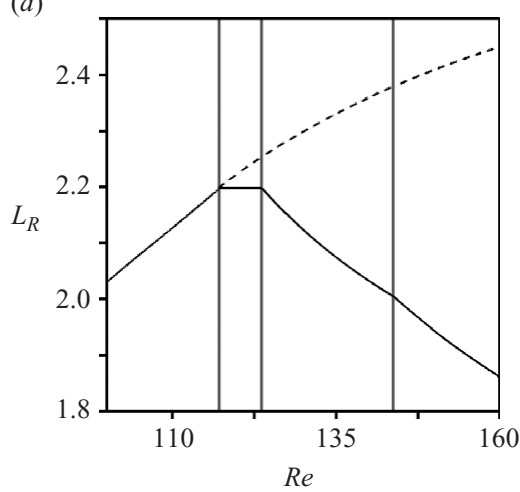

(b)

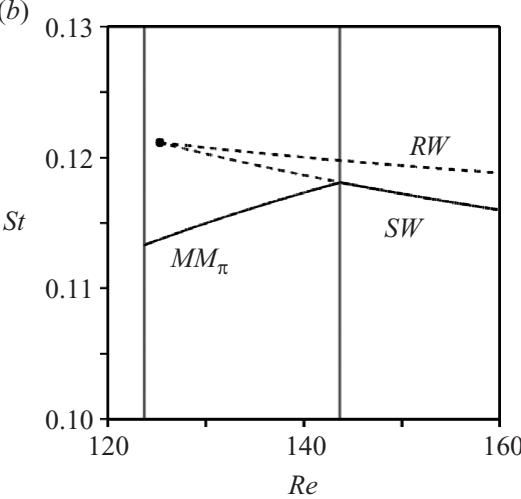

FiguRE 17. (a) Recirculation length $L_{r}$ for the mean flow as a function of the Reynolds number. (b) Strouhal number $S t$ of the unsteady flow as a function of the Reynolds number.

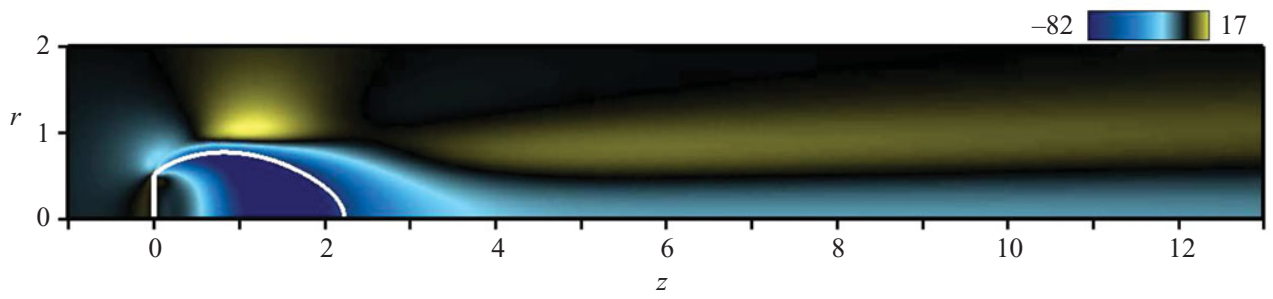

FIGURE 18. Axial velocity component $\hat{w}_{\delta}^{2}$ of the base flow modification owing to the variation of the Reynolds number. The solid line in the flow indicates the separatrix of the recirculation zone.

\subsection{Recirculation length and Strouhal number evolution}

We investigate now the impact of the bifurcation sequence on the recirculation length and frequency of the bifurcated flow. Figure 17( $a$ ) shows the evolution of the recirculation length as a function of the Reynolds number. The vertical grey lines stand for the different bifurcation thresholds. The solid line corresponds to that of the mean flow obtained by time and azimuthal average, given in the present formalism by the axisymmetric stationary solution up to the second order

$$
\boldsymbol{q}=\boldsymbol{q}^{\mathbf{0}}+\epsilon^{2}\left(\delta \hat{\boldsymbol{q}}_{\delta}^{\mathbf{2}}+|A|^{2} \hat{\boldsymbol{q}}_{\boldsymbol{A} \boldsymbol{A}^{*}}^{\mathbf{2}}+\left|B^{+}\right|^{2} \hat{\boldsymbol{q}}_{\boldsymbol{B}^{+} \boldsymbol{B}^{+*}}^{\mathbf{2}}+\left|B^{-}\right|^{2} \hat{\boldsymbol{q}}_{\boldsymbol{B}^{-} \boldsymbol{B}^{-*}}^{\mathbf{2}}\right),
$$

and the dashed line corresponds to that of the axisymmetric base flow obtained as $\boldsymbol{q}^{\mathbf{0}}+\epsilon^{2} \delta \hat{\boldsymbol{q}}_{\delta}^{\mathbf{2}}$. The results sketched in this figure show that increasing the Reynolds number yields an increase in the recirculation length of the base flow. Figure 18 shows the axial velocity component $\hat{w}_{\delta}^{2}$ of the base flow modification owing to the variation of the Reynolds number. It can be seen that the increase in the recirculation length is due to $\hat{w}_{\delta}^{2}$ being negative in the wake. It is possible to note that on the $S S$ branch, this effect is exactly counterbalanced by the positive values of $\hat{w}_{A A^{*}}^{2}$, so that the overall recirculation length remains almost constant between $R e_{s}$ and $R e_{\pi}^{A}$. For Reynolds numbers larger than $R e_{\pi}^{A}$, the positive values of $\hat{w}_{A A^{*}}^{2}$ and $\hat{w}_{B^{ \pm} B_{ \pm^{ \pm *}}}^{2}$ in the wake (not shown here) become dominant and the overall recirculation length continuously decreases down to 1.8 disk diameters. Note that in the case of the wake past a circular cylinder, a similar decrease of the recirculation length as the Reynolds number is increased above the critical value $R e=47$ has been shown to arise due 
to the strong mean flow correction induced by the existence of an unstable mode (Zielinska et al. 1997).

Another consequence of this successive bifurcation scenario is the prediction of the frequency at the onset of unsteadiness. The frequency of the bifurcated flow is given by $\omega=\omega_{0}+\epsilon^{2} \tilde{\omega}_{n l}$ where $\tilde{\omega}_{n l}$ is the second-order nonlinear correction obtained from equations (4.23b) and $(4.25 d)$ as $\dot{\phi}_{\mathrm{B}}^{ \pm}=\epsilon^{2} \tilde{\omega}_{n l}$. Figure $17(b)$ shows the evolution of the Strouhal number $S t=D \omega /\left(2 \pi U_{\infty}\right)$ as a function of the Reynolds number. The continuous line represents the frequency of the selected $M M_{\pi}$ and $S W$ modes, and the dashed lines represent the frequency of the unstable $S W$ and $R W$ modes. As already commented, the bifurcation from the nonlinear $S S$ branch to the $M M_{\pi}$ solution occurs earlier than that from the axisymmetric state to the oscillating modes. The remarkable feature is that the Strouhal number at the bifurcation is lower than that predicted solely by the stability analysis of the axisymmetric state by approximately $10 \%$ (0.11 at $R e_{\pi}^{A}$ on the $M M_{\pi}$ branch and 0.12 at $R e_{c}^{B}$ on the $S W$ branch). This provides a simple explanation for the discrepancy between the shedding frequency predicted by the linear stability theory and by the full Navier-Stokes computations, as mentioned in Fabre et al. (2008).

\section{Influence of external noise on the sequence of bifurcations}

In experimental set-ups, the geometry of the apparatus, and in particular supporting devices, induce steady perturbations that may affect the bifurcation properties. In this section, we investigate the sensitivity of the theoretical bifurcation diagram shown in figure 8 to such perturbations. We consider that the imperfections act as a smallamplitude steady forcing term in the Navier-Stokes equations, that now read

$$
\nabla \cdot \boldsymbol{u}=0, \quad \partial_{t} \boldsymbol{u}+\nabla \boldsymbol{u} \cdot \boldsymbol{u}+\nabla p-\frac{1}{R e} \nabla^{2} \boldsymbol{u}=\boldsymbol{f}_{s},
$$

where $\boldsymbol{f}_{s}$ is the steady forcing term, which, anticipating on the dominant balance, may be taken of order $\epsilon^{3}$. The associated disturbance $\boldsymbol{f}_{s}$ can then be decomposed into the superposition of perturbations of various azimuthal wavenumbers:

$$
\boldsymbol{f}_{s}=\epsilon^{3} \sum_{m=0}^{\infty} \hat{\boldsymbol{f}}_{\boldsymbol{s}}^{\boldsymbol{m}}(r, z) e^{\mathrm{i} m \theta}
$$

Being assumed of order three in $\epsilon$, this steady external forcing directly adds on to the third order forcing term in expansion (4.13). Among all contributions arising from $\boldsymbol{f}_{s}$, only $\hat{\boldsymbol{f}}_{s}^{\mathbf{1}}$ is resonant and adds the new term

$$
\alpha_{f}=\left\langle\hat{\boldsymbol{q}}_{A}^{1 \dagger}, \hat{\boldsymbol{f}}_{s}^{\mathbf{1}}\right\rangle
$$

to the amplitude equation $(4.14 a)$ for the stationary mode $\hat{\boldsymbol{q}}_{A}^{\mathbf{1}}$, so that the perturbed system reads

$$
\begin{aligned}
\dot{A} & =\epsilon^{2}\left(\alpha_{f}+\tilde{\lambda}_{A} A-\tilde{\mu}_{A} A|A|^{2}-\tilde{v}_{A} A\left|B^{+}\right|^{2}-\tilde{v}_{A}^{*} A\left|B^{-}\right|^{2}-\tilde{\chi}_{A} B^{+} B^{-*} A^{*}\right), \\
\dot{B}^{+} & =\epsilon^{2}\left(\tilde{\lambda}_{B}-\tilde{\mu}_{B} B^{+}\left|B^{+}\right|^{2}-\tilde{v}_{B} B^{+}\left|B^{-}\right|^{2}-\tilde{\eta}_{B} B^{+}|A|^{2}-\tilde{\chi}_{B} B^{-} A^{2}\right), \\
\dot{B}^{-} & =\epsilon^{2}\left(\tilde{\lambda}_{B}-\tilde{\mu}_{B} B^{-}\left|B^{-}\right|^{2}-\tilde{v}_{B} B^{-}\left|B^{+}\right|^{2}-\tilde{\eta}_{B} B^{-}|A|^{2}-\tilde{\chi}_{B} B^{+} A^{* 2}\right) .
\end{aligned}
$$

Interestingly, for $\left|B^{ \pm}\right|=0$, the imaginary part of $(5.4 a)$ reduces to

$$
\dot{\phi}_{\mathrm{A}}=\frac{\left|\alpha_{f}\right|}{|A|} \sin \left(\phi_{f}-\phi_{\mathrm{A}}\right),
$$




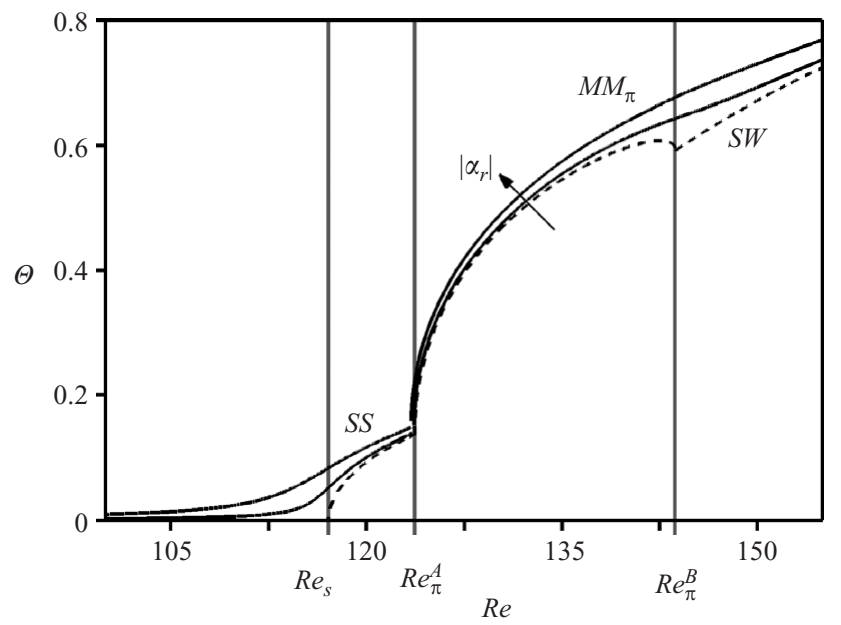

FIgURE 19. Bifurcation diagram for various forcing amplitudes $\left|\alpha_{f}\right|$.

where $\phi_{f}=\arg \left(\alpha_{f}\right)$, so that a steady solution requires that $\phi_{\mathrm{A}}=\phi_{f} \pm \pi$. The plane corresponding to the symmetry lost at the first bifurcation threshold is no more arbitrary, but is selected by the forcing term, even if of very small amplitude (order $\left.\epsilon^{3}\right)$. Figure 19 shows the perturbed bifurcation diagrams for $\left|\alpha_{f}\right|=2.5 \times 10^{-2}$ and $10^{-1}$. The first steady bifurcation degenerates into an imperfect bifurcation, so that the amplitude $A$ of the steady mode $\hat{\boldsymbol{q}}_{A}^{\mathbf{1}}$ is non-zero even at Reynolds numbers lower than the first threshold. The Hopf bifurcation from the $S S$ to the $M M_{\pi}$ branch is preserved and occurs at a Reynolds number very close to the threshold $\operatorname{Re}_{\pi}^{A}=123.7$ found in the unperturbed case: we obtain $R e_{\pi}^{A^{*}}=123.6$ for $\left|\alpha_{f}\right|=2.5 \times 10^{-2}$ and 123.4 for $\left|\alpha_{f}\right|=10^{-1}$. The bifurcation from the $M M_{\pi}$ to the $S W$ branch also degenerates into an imperfect bifurcation, so that the flow ultimately remains three-dimensional without recovering its lost reflectional symmetry, even for very low forcing amplitudes.

\section{Conclusion}

In this study, we have considered the nonlinear dynamics of the wake of a circular disk placed normal to a uniform flow. The performed linear stability analysis agrees with the results of Natarajan \& Acrivos (1993). The first destabilization of the axisymmetric steady flow occurs for a stationary global mode of azimuthal wavenumber $m=1$ and the second destabilization occurs for two oscillating global modes of azimuthal wavenumbers $m= \pm 1$. Since the critical Reynolds numbers for these three bifurcating modes are close to one another, we have assumed that the nonlinear dynamics close to these threshold Reynolds numbers could be described by the slow manifold spanned by the three destabilizing eigenmodes. To derive rigorously the normal form governing the dynamics in the slow manifold, a multiple time scale expansion has been carried out. In this procedure, the first-order solution is made of the three modes with unknown amplitudes. The second order is decomposed into the base flow modification owing to the variation in the Reynolds number and harmonics generated by the nonlinear self-interaction of the first-order solution. At third order, resonant terms are generated and the resulting solvability conditions impose the nonlinear equations that must be satisfied by the unknown amplitudes, the so-called normal form. Coefficients of the normal form have been systematically computed. 


\begin{tabular}{lrrr} 
& \multicolumn{1}{c}{$\sigma_{A}$} & \multicolumn{1}{c}{$\sigma_{B}$} & $\omega_{B}$ \\
$M_{1}$ & $5.3 \times 10^{-6}$ & $4.7 \times 10^{-6}$ & 0.7604 \\
$M_{2}$ & $-9.9 \times 10^{-5}$ & $-9.0 \times 10^{-5}$ & 0.7605 \\
$M_{3}$ & $-5.5 \times 10^{-5}$ & $-4.0 \times 10^{-5}$ & 0.7604 \\
$M_{4}$ & $1.3 \times 10^{-5}$ & $1.6 \times 10^{-5}$ & 0.7605 \\
$M_{5}$ & $8.9 \times 10^{-5}$ & $6.6 \times 10^{-5}$ & 0.7604
\end{tabular}

TABLE 4. Dependence of the eigenvalues on the different meshes characterized in table 1. The real eigenvalue $\sigma_{A}$ is computed at $R e_{c}^{A}=116.9$ and the complex eigenvalue $\sigma_{B}+\mathrm{i} \omega_{B}$ is computed at $R e_{c}^{B}=125.3$.

They are given by the scalar product between a resonant forcing term arising at the third order and the adjoint of the forced mode. Owing to the convective nonnormality of the linearized Navier-Stokes operator, we have shown that strikingly, the region where all nonlinear interactions take place is located within the recirculating bubble. Analysing the dynamics resulting from the normal form, we find that the wake undergoes a first bifurcation for $R e=117.1$, where the axisymmetry is lost but the time invariance is preserved, leading to a three-dimensional steady state with a reflectional symmetry. A Hopf bifurcation then occurs for $R e=123.7$, where both the remaining reflectional symmetry and the time invariance are broken, leading to a fully three-dimensional periodic state. A third bifurcation then occurs for $R e=143.7$, where the flow remains unsteady, but recovers the reflectional symmetry normal to that lost at the first bifurcation threshold. The non-trivial bifurcation sequence involving nonlinear interactions between unstable modes of the axisymmetric base flow agrees remarkably with the recent observations made by Fabre et al. (2008) using DNS. These authors have reported three successive bifurcations at Reynolds numbers $R e \simeq 115$, 121 and 140, giving rise to bifurcated states with symmetry features identical to the ones found in this study. Furthermore, the frequency they have observed for the mixed state is lower than the frequency of the oscillating mode at threshold, an effect that results from the existence of the $M M_{\pi}$ mixed mode in the present study. This suggests that the three-dimensional dynamics of the whole system is efficiently captured using a reduced order model based on the destabilization of the axisymmetric steady state.

\section{Appendix A. Sensitivity of the results to mesh spacing}

The eigenvalues, as well as the nonlinear coefficients of normal form (4.14) have been calculated for the five meshes $M_{1}-M_{5}$, differing by the location of the external boundaries and by the vertex densities. Results are given in table 4 for the values of the linear growth rate $\sigma$ and frequency $\omega$ at threshold, for both eigenmodes $\hat{\boldsymbol{q}}_{A}^{1}$ and $\hat{\boldsymbol{q}}_{\boldsymbol{B}^{ \pm}}{ }^{1}$. Even for the coarser mesh $M_{5}$, the growth rate is zero down to the fourth digit, and the frequency of the oscillating mode is converged down to the third digit. On table 5, the coupling coefficients of normal form (4.14) are compared for the different meshes. Even for this very involved computations, coefficients are converged down to the third digit. Therefore, we can conclude that the present work is precise down to better than $1 \%$. Note that this is the numerical precision but not the convergence of the asymptotic expansion, whose precision increases as $\left|R e-R e_{c}\right|$ decreases. 


\begin{tabular}{ccccccccccccc}
\hline & & & & & & & & & & & \\
& $\tilde{\mu}_{A}$ & $\tilde{v}_{A r}$ & $\tilde{v}_{A i}$ & $\tilde{\chi}_{A}$ & $\tilde{\mu}_{B r}$ & $\tilde{\mu}_{B i}$ & $\tilde{v}_{B r}$ & $\tilde{v}_{B i}$ & $\tilde{\eta}_{B r}$ & $\tilde{\eta}_{B i}$ & $\tilde{\chi}_{B r}$ & $\tilde{\chi}_{B i}$ \\
$M_{1}$ & 3.11 & 6.88 & -1.11 & 4.57 & 2.42 & 0.0321 & 3.13 & -0.816 & 0.955 & -3.47 & 1.62 & -1.36 \\
$M_{2}$ & 3.11 & 6.88 & -1.11 & 4.57 & 2.42 & 0.0332 & 3.13 & -0.814 & 0.955 & -3.47 & 1.62 & -1.36 \\
$M_{3}$ & 3.11 & 6.88 & -1.11 & 4.57 & 2.42 & 0.0329 & 3.13 & -0.815 & 0.955 & -3.47 & 1.62 & -1.36 \\
$M_{4}$ & 3.11 & 6.88 & -1.11 & 4.57 & 2.42 & 0.0320 & 3.13 & -0.817 & 0.955 & -3.47 & 1.62 & -1.36 \\
$M_{5}$ & 3.11 & 6.88 & -1.11 & 4.56 & 2.42 & 0.0310 & 3.13 & -0.807 & 0.962 & -3.47 & 1.62 & -1.36
\end{tabular}

TABLE 5. Coefficient values of normal form (4.14) obtained for the different meshes characterized in table 1 .

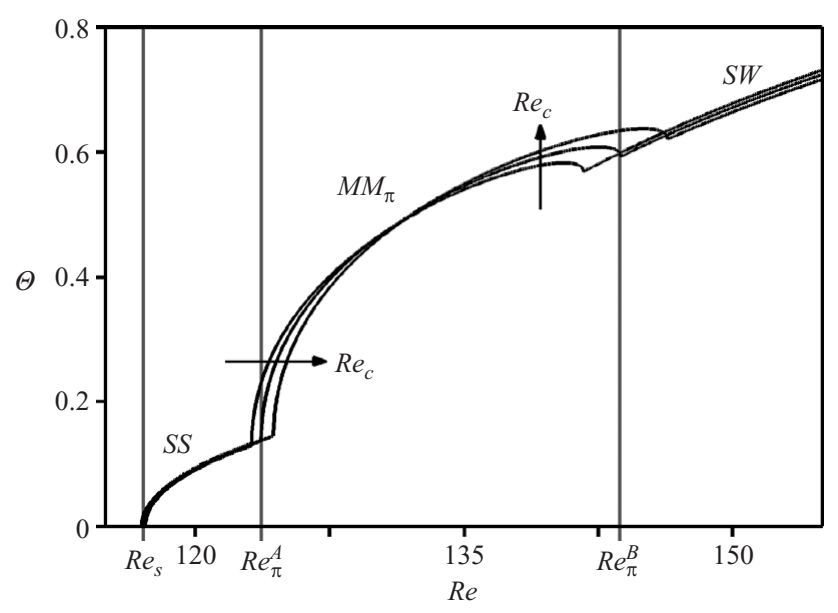

FIGURE 20. Bifurcation diagram obtained for different choices of the reference Reynolds number $\operatorname{Re}_{c}=116.9,121.1$ and 125.3.

\section{Appendix B. Comparison of bifurcation diagrams obtained with various choices of the reference Reynolds number}

Figure 20 presents the bifurcation diagram obtained for different choices of the reference Reynolds number $R e_{c}$. Computations for $R e_{c}=116.9$ and $R e_{c}=125.3$ yield slightly different nonlinear coefficients, resulting in diagrams in good agreement with each other (see figure 20). It can be seen that the choice of $R e_{c}$ has no significant impact on the dynamics, both in terms of mode selection and symmetry breaking. We find a small effect on the bifurcation thresholds related to the $M M_{\pi}$ branch: the second threshold $R e_{\pi}^{A}$ varies by less that $0.5 \%$, from 123.3 to 124.4 , and the third threshold $R e_{\pi}^{B}$ varies by approximately $3 \%$, from 140.9 to 146.7 . This sensitivity is remarkably small since the expansion procedure is meant for small departures from threshold, which is no more the case at the third bifurcation threshold $\operatorname{Re}_{\pi}^{B}$.

\section{Appendix C. Expression of the complex differential operators}

For a normal mode $\hat{\boldsymbol{a}}$ of azimuthal wavenumber $m$, the gradient operator and the velocity gradient tensor respectively read

$$
\nabla_{\boldsymbol{m}}=\left(\begin{array}{c}
\partial_{r} \\
\frac{\mathrm{i} m}{r} \\
\partial_{z}
\end{array}\right), \quad \nabla_{\boldsymbol{m}} \hat{\boldsymbol{a}}=\left(\begin{array}{ccc}
\partial_{r} \hat{u} & \frac{\mathrm{i} m}{r} \hat{u}-\frac{1}{r} \hat{v} & \partial_{z} \hat{u} \\
\partial_{r} \hat{v} & \frac{\mathrm{i} m}{r} \hat{v}+\frac{1}{r} \hat{u} & \partial_{z} \hat{v} \\
\partial_{r} \hat{w} & \frac{\mathrm{i} m}{r} \hat{w} & \partial_{z} \hat{w}
\end{array}\right) .
$$


For a couple of normal modes $\hat{\boldsymbol{a}}$ and $\hat{\boldsymbol{b}}$ of respective azimuthal wavenumbers $m$ and $n$, the general form of the linearized advection operator $\mathscr{C}_{m, n}(\hat{\boldsymbol{a}}, \hat{\boldsymbol{b}})$ therefore reads

$$
\mathscr{C}_{m, n}(\hat{\boldsymbol{a}}, \hat{\boldsymbol{b}})=\left(\begin{array}{c}
\left.\hat{u}_{b} \partial_{r} \hat{u}_{a}+\hat{u}_{a} \partial_{r} \hat{u}_{b}+\frac{\mathrm{i}}{r}\left(m \hat{v}_{b} \hat{u}_{a}+n \hat{v}_{a} \hat{u}_{b}\right)-\frac{2}{r} \hat{v}_{b} \hat{v}_{a}+\hat{w}_{b} \partial_{z} \hat{u}_{a}+\hat{w}_{a} \partial_{z} \hat{u}_{b}\right) \\
\hat{u}_{r} \hat{v}_{a}+\hat{u}_{a} \partial_{r} \hat{v}_{b}+\frac{\mathrm{i}}{r}(m+n) \hat{v}_{b} \hat{v}_{a}+\frac{1}{r}\left(\hat{v}_{b} \hat{u}_{a}+\hat{v}_{a} \hat{u}_{b}\right)+\hat{w}_{b} \partial_{z} \hat{v}_{a}+\hat{w}_{a} \partial_{z} \hat{v}_{b} \\
\hat{u}_{b} \partial_{r} \hat{w}_{a}+\hat{u}_{a} \partial_{r} \hat{w}_{b}+\frac{\mathrm{i}}{r}\left(m \hat{v}_{b} \hat{w}_{a}+n \hat{v}_{a} \hat{w}_{b}\right)+\hat{w}_{b} \partial_{z} \hat{w}_{a}+\hat{w}_{a} \partial_{z} \hat{w}_{b}
\end{array}\right) .
$$

The linearized advection operator used in the order one problem (3.6) now reads

$$
\mathscr{C}_{m, 0}\left(\hat{\boldsymbol{u}}^{\mathbf{1}}, \boldsymbol{u}^{\mathbf{0}}\right)=\left(\begin{array}{c}
\left.\hat{u}^{0} \partial_{r} \hat{u}^{1}+\hat{u}^{1} \partial_{r} \hat{u}^{0}+\frac{i}{r} m \hat{v}^{1} \hat{u}^{0}+\hat{w}^{0} \partial_{z} \hat{u}^{1}+\hat{w}^{1} \partial_{z} \hat{u}^{0}\right) \\
\hat{u}^{0} \partial_{r} \hat{v}^{1}+\frac{1}{r} \hat{v}^{1} \hat{u}^{0}+\hat{w}^{0} \partial_{z} \hat{v}^{1} \\
\hat{u}^{0} \partial_{r} \hat{w}^{1}+\hat{u}^{1} \partial_{r} \hat{w}^{0}+\frac{i}{r} m \hat{v}^{1} \hat{w}^{0}+\hat{w}^{0} \partial_{z} \hat{w}^{1}+\hat{w}^{1} \partial_{z} \hat{w}^{0}
\end{array}\right)
$$

Finally, the adjoint linearized advection operator used in the adjoint problem (3.8) reads

$$
\mathscr{C}_{m, 0}^{\dagger}\left(\hat{\boldsymbol{u}}^{\mathbf{1}}, \boldsymbol{u}^{\mathbf{0}}\right)=\left(\begin{array}{c}
-u^{0} \partial_{r} \hat{u}^{1}+\hat{u}^{1} \partial_{r} u^{0}-w^{0} \partial_{z} \hat{u}^{1}+\hat{w}^{1} \partial_{r} w^{0} \\
-u^{0} \partial_{r} \hat{v}^{1}+\frac{1}{r} \hat{v}^{1} u^{0}-w^{0} \partial_{z} \hat{v}^{1} \\
-u^{0} \partial_{r} \hat{w}^{1}+\hat{u}^{1} \partial_{z} u^{0}-w^{0} \partial_{z} \hat{w}^{1}+\hat{w}^{1} \partial_{z} w^{0}
\end{array}\right)
$$

\section{REFERENCES}

BARKLEY, D. 2006 Linear analysis of the cylinder wake mean flow. Europhys. Lett. 75, 750-756.

Barkley, D., Gomes, M. G. M. \& Henderson, R. D. 2002 Three-dimensional instability in flow over a backward-facing step. J. Fluid Mech. 473, 167-190.

Chомaz, J.-M. 2005 Global instabilities in spatially developing flows: non-normality and nonlinearity. Annu. Rev. Fluid. Mech. 37, 357-392.

Chomaz, J.-M., Huerre, P. \& Redekopp, L. G. 1990 The effect of nonlinearity and forcing on global modes. In Proceedings of the Conf. on New Trends in Nonlinear Dynamics and PatternForming Phenomena: The Geometry of Nonequilibrium (ed. P. Coulet \& P. Huerre), vol. 237, pp. 259-274. NATO ASI Series B, Plenum.

Chossat, P. \& Iooss, G. 1994 The Couette-Taylor problem. Appl. Math. Sci. 102.

Crawford, J. D., Golubitsky, M. \& Langford, W. F. 1988 Modulated rotating waves in $O(2)$ mode interactions. Dyn. Stab. Syst. 3, 159-175.

Crawford, J. D. \& KNobloch, E. 1991 Symmetry, symmetry-breaking bifurcations in fluid dynamics. Annu. Rev. Fluid. Mech. 23, 341-387.

Cross, M. C. 1986 Traveling and standing waves in binary-fluid convection in finite geometries. Phys. Rev. Lett. 57, 2935-2938.

DAvis, T. A. 2004 A column pre-ordering strategy for the unsymmetric-pattern multifrontal method. ACM Trans. Math. Softw. 30 (2), 165-195.

Davis, T. A. \& DufF, I. S. 1997 An unsymmetric-pattern multifrontal method for sparse LU factorization. SIAM J. Matrix Anal. Appl. 18 (1), 140-158.

Ding, Y. \& KawahaRA, M. 1999 Three-dimensional linear stability analysis of incompressible viscous flows using finite element method. Intl J. Numer. Methods Fluids 31, 451-479.

Dušek, J., Le Gal, P. \& Fraunie, P. 1994 A numerical and theoretical study of the first Hopf bifurcation in a cylinder wake. J. Fluid Mech. 264, 59-80.

Ehrenstein, U. \& Gallaire, F. 2005 On two-dimensional temporal modes in spatially evolving open flows: the flat-plate boundary layer. J. Fluid Mech. 536, 209-218.

Fabre, D., Auguste, F. \& Magnaudet, J. 2008 Bifurcations and symmetry breaking in the wake of axisymmetric bodies. Phys. Fluids 20 (5), 051702 1-4.

FriedRICHS, K. O. 1973 Spectral Theory of Operators in Hilbert Space. Springer. 
Golubitsky, M. \& LANGFORD, W. 1988 Pattern formation and bistability in flow between counterrotating cylinders. Physica D 32, 362-392.

Golubitsky, M. \& Stewart, I. 1985 Hopf bifurcation in the presence of symmetry. Arch. Ration. Mech. Anal. 87, 107-165.

Golubitsky, M., Stewart, I. \& Schaeffer, D. 1988 Singularities and Groups in Bifurcation Theory Vol. II - Applied Mathematical Sciences. Springer.

Gumowski, K., Miedzik, J., Goujon-Durand, S., Jenffer, P. \& Wesfreid, J. E. 2008 Transition to a time-dependent state of fluid flow in the wake of a sphere. Phys. Rev. E 77, 055308, 1-4.

JACKSON, C. P. 1987 A finite-element study of the onset of vortex shedding in flow past variously shaped bodies. J. Fluid Mech. 182, 23-45.

NAtarajan, R. \& ACrivos, A. 1993 The instability of the steady flow past spheres and disks. J. Fluid Mech. 254, 323-344.

Pier, B. 2008 Local and global instabilities in the wake of a sphere. J. Fluid Mech. 603, 39-61.

Provansal, M., Mathis, C. \& Boyer, L. 1987 Bénard von-Kármán instability: transient and forced regimes. J. Fluid Mech. 182, 1-22.

Schmid, P. J. \& Henningson, D. S. 2001 Stability and Transition in Shear Flows. Springer.

SipP, D. \& LEBEDEV, A. 2007 Global stability of base and mean flows: a general approach and its applications to cylinder and open cavity flows. J. Fluid Mech. 593, 333-358.

Thompson, M. C., Leweke, T. \& Provansal, M. 2001 Kinematics and dynamics of sphere wake transition. J. Fluid Struct. 15, 575-585.

ZEBIB, A. 1987 Stability of a viscous flow past a circular cylindar. J. Engng Math. 21, 155-165.

Zielinska, B. J. A., Goujon-Durand, S., Dušek, J. \& Wesfreid, J. E. 1997 Strongly nonlinear effect in unstable wakes. Phys. Rev. Lett. 79, 3893-3896. 